\title{
LARGE SOLUTIONS FOR A SYSTEM OF ELLIPTIC EQUATIONS ARISING FROM FLUID DYNAMICS*
}

\author{
J. I. DÍAZ ${ }^{\dagger}$, M. LAZZO ${ }^{\ddagger}$ AND P. G. SCHMIDT S $^{\S}$
}

Abstract. This paper is concerned with the elliptic system

$$
\Delta v=\phi, \quad \Delta \phi=|\nabla v|^{2}
$$

posed in a bounded domain $\Omega \subset \mathbb{R}^{N}, N \in \mathbb{N}$. Specifically, we are interested in the existence and uniqueness or multiplicity of "large solutions," that is, classical solutions of (0.1) that approach infinity at the boundary of $\Omega$. Assuming that $\Omega$ is a ball, we prove that the system (0.1) has a unique radially symmetric and nonnegative large solution with $v(0)=0$ (obviously, $v$ is determined only up to an additive constant). Moreover, if the space dimension $N$ is sufficiently small, there exists exactly one additional radially symmetric large solution with $v(0)=0$ (which, of course, fails to be nonnegative). We also study the asymptotic behavior of these solutions near the boundary of $\Omega$ and determine the exact blow-up rates; those are the same for all radial large solutions and independent of the space dimension. Our investigation is motivated by a problem in fluid dynamics. Under certain assumptions, the unidirectional flow of a viscous, heat-conducting fluid is governed by a pair of parabolic equations of the form

$$
v_{t}-\Delta v=\theta, \quad \theta_{t}-\Delta \theta=|\nabla v|^{2},
$$

where $v$ and $\theta$ represent the fluid velocity and temperature, respectively. The system (0.1), with $\phi=-\theta$, is the stationary version of $(0.2)$.

Key words. elliptic system, boundary blow-up, large solutions, radial solutions, existence and multiplicity, asymptotic behavior

AMS subject classifications. 35J60, 35J55, 35Q35

DOI. $10.1137 /$ S0036141004443555

1. Introduction and main results. This paper is a contribution to the study of "explosive behavior" in certain systems of elliptic and parabolic PDEs. Our investigation is motivated by a question regarding the dynamics of a viscous, heat-conducting fluid.

In general, the flow of such a fluid is governed by a system of balance equations for momentum, mass, and energy. Under the assumptions of the so-called Boussinesq approximation, this system reduces to the Navier-Stokes equations for an incompressible fluid, along with a heat equation; the equations are nonlinearly coupled through the buoyancy force and viscous heating. If viscous heating (that is, the production of heat due to internal friction) is neglected, the resulting boundary and initial-boundary value problems are well posed in the same sense as for the classical Navier-Stokes equations without thermal coupling; but if viscous heating is taken into account, well-posedness is an open question. In fact, we conjecture that the solutions,

*Received by the editors May 6, 2004; accepted for publication (in revised form) January 19, 2005; published electronically October 14, 2005.

http://www.siam.org/journals/sima/37-2/44355.html

${ }^{\dagger}$ Departamento de Matemática Aplicada, Universidad Complutense de Madrid, Avenida Complutense s/n, 28040 Madrid, Spain (ji_diaz@mat.ucm.es). The research of this author was partially supported by project REN2003-0223-C03 of the DGISGPI (Spain) and project RTN HPRN-CT2002-00274 of the European Union.

${ }^{\ddagger}$ Dipartimento di Matematica, Università degli Studi di Bari, via Orabona 4, 70125 Bari, Italy (lazzo@dm.uniba.it).

$\S$ Department of Mathematics and Statistics, Parker Hall, Auburn University, AL 36849-5310 (pgs@cam.auburn.edu). 
in this case, may exhibit "explosive behavior." Such behavior would have implications for the viability of the Boussinesq approximation in situations where viscous heating cannot be neglected.

To address this issue, we are studying a simple prototype problem, which can be physically justified by considering a unidirectional flow, independent of distance in the flow direction:

$$
v_{t}-\Delta v=\theta, \quad \theta_{t}-\Delta \theta=|\nabla v|^{2} .
$$

Here, $v$ (the velocity) and $\theta$ (the temperature) are scalar functions of time $t$ and position $x$; the spatial variable $x$ varies over a bounded domain $\Omega \subset \mathbb{R}^{N}$ with $N \in \mathbb{N}$ ( $N=2$ in the physically relevant case, where $\Omega$ is the cross-section of the flow channel). The source terms $\theta$ and $|\nabla v|^{2}$ represent the buoyancy force and viscous heating, respectively. The system (1.1) must be supplemented by suitable initial conditions at time $t=0$ and boundary conditions on the boundary $\partial \Omega$ of the domain $\Omega$ (for example, a homogeneous Dirichlet condition for $v$ and a homogeneous Neumann condition for $\theta$ if the walls of the flow channel are impermeable and thermally insulated).

Note that we cannot hope to find weak solutions of the resulting initial-boundary value problem in the usual Hilbert-space setting: if $v$ takes values in $H^{1}(\Omega)$, then the right-hand side of the second equation in (1.1) maps, a priori, only into $L^{1}(\Omega)$. However, local-in-time existence and uniqueness of a strong solution can be established by means of semigroup theory in a suitable $L^{p}$-space setting. We conjecture that this solution may blow up in finite time, in the sense that a suitable norm of $(v, \theta)$ approaches infinity as $t \rightarrow T^{-}$, for some $T>0$. Preliminary analytical and numerical results for the parabolic problem will appear in a forthcoming publication.

In the present paper, we consider the stationary version of (1.1), that is, the elliptic system

$$
-\Delta v=\theta, \quad-\Delta \theta=|\nabla v|^{2}
$$

posed in a domain $\Omega \subset \mathbb{R}^{N}$ with $N \in \mathbb{N}$. Specifically, we are interested in the possibility of "boundary blow-up," that is, the existence of classical solutions $(v, \theta)$ of (1.2) with $|(v(x), \theta(x))| \rightarrow \infty$ as $\operatorname{dist}(x, \partial \Omega) \rightarrow 0$ (so-called large solutions). Note that the $\theta$-component of any solution of (1.2) is superharmonic in $\Omega$ and thus cannot approach $\infty$ at the boundary (maximum principle); for a similar reason, $v$ and $\theta$ cannot simultaneously approach $-\infty$ at the boundary. We therefore expect any large solution $(v, \theta)$ of $(1.2)$ to satisfy $v(x) \rightarrow \infty$ and $\theta(x) \rightarrow-\infty$ as $\operatorname{dist}(x, \partial \Omega) \rightarrow 0$.

The preceding observation implies that large solutions of (1.2) cannot be expected to describe the asymptotics of explosive solutions of the parabolic system (1.1). Assuming, for example, that the temperature $\theta$ in (1.1) satisfies a homogeneous Neumann boundary condition on $\partial \Omega$, the temperature minimum is a nondecreasing function of time (parabolic maximum principle); thus, $\theta$ cannot approach $-\infty$ at the boundary. Nevertheless, boundary blow-up in the elliptic system (1.2) would have implications for the dynamics of the parabolic system (1.1) and its controllability. For example, large solutions of (1.2) may be used to construct "universal distributed bounds" (that is, interior bounds independent of the boundary data) for solutions of associated initial-boundary value problems and their steady states. We refer the reader to $[3,6,7$, $14,23]$ and the references therein for similar arguments and applications in the context of other semilinear or quasilinear parabolic problems with superlinear nonlinearities.

Henceforth, we assume that $\Omega$ is a ball in $\mathbb{R}^{N}$, centered at the origin; that is, $\Omega=B_{R}^{N}(0)$ for some $R>0$. For convenience, we introduce the function $\phi=-\theta$ and 
seek radially symmetric large solutions of the problem

$$
\Delta v=\phi, \quad \Delta \phi=|\nabla v|^{2} \quad \text { in } B_{R}^{N}(0),
$$

that is, radial solutions $(v, \phi)$ with $|(v(x), \phi(x))| \rightarrow \infty$ as $|x| \rightarrow R^{-}$.

REMARK 1.1. The problem (1.3) has a scaling property that we will exploit repeatedly. Suppose $\left(v_{1}, \phi_{1}\right)$ is a (large) solution of (1.3) with $R=R_{1}$. For $\lambda \in(0, \infty)$, let $R_{\lambda}:=\lambda^{-1} R_{1}$. For $x \in B_{R_{\lambda}}^{N}(0)$, define

$$
v_{\lambda}(x):=\lambda^{2} v_{1}(\lambda x), \quad \phi_{\lambda}(x):=\lambda^{4} \phi_{1}(\lambda x) .
$$

Then $\left(v_{\lambda}, \phi_{\lambda}\right)$ is a (large) solution of (1.3) with $R=R_{\lambda}$.

REMARK 1.2. If $(v, \phi)$ is a (large) solution of $(1.3)$, then so is $(v+c, \phi)$, for any constant $c \in \mathbb{R}$. Thus, we may restrict attention to solutions with $v(0)=0$.

We will now state our main results, the first of which guarantees the existence of a unique (up to a shift in $v$ ) radially symmetric and nonnegative large solution for any space dimension.

THEOREM 1.3. For every $N \in \mathbb{N}$ and $R>0$, the problem (1.3) has a unique radially symmetric large solution $(v, \phi)$ with $v(0)=0$ and $\phi(0)>0$. Both components of this solution are increasing functions of the radial variable $r$.

If the space dimension is sufficiently small, there exists exactly one additional radially symmetric large solution with $v(0)=0$, which, of course, fails to be nonnegative.

TheOREm 1.4. For every $N \in \mathbb{N}$ with $N \leq 10$ and every $R>0$, the problem (1.3) has a unique radially symmetric large solution $(v, \phi)$ with $v(0)=0$ and $\phi(0)<0$. The $\phi$-component of this solution is an increasing function of the radial variable $r$, while the $v$-component is decreasing to a negative minimum and increasing thereafter.

Let us note that the bound on $N$ in the above result is not sharp. In fact, based on numerical evidence (see Remarks 3.5 and 4.4), we conjecture that the solution of Theorem 1.4 exists if and only if $N \leq 14$.

With regard to asymptotic behavior, we find that, as expected, both components of a large solution approach infinity at the boundary, and we determine the exact blow-up rates; those are the same for all radially symmetric large solutions and independent of the space dimension. Here and in what follows, we write $f(x) \sim g(x)$ if the mappings $f, g: B_{R}^{N}(0) \rightarrow \mathbb{R}$ satisfy $f(x) / g(x) \rightarrow 1$ as $|x| \rightarrow R^{-}$.

THEOREM 1.5. Let $(v, \phi)$ be any radially symmetric large solution of (1.3), for a given $N \in \mathbb{N}$ and $R>0$. Then, as $|x| \rightarrow R^{-}$,

$$
v(x) \sim \frac{30}{(R-|x|)^{2}} \quad \text { and } \quad \phi(x) \sim \frac{180}{(R-|x|)^{4}} .
$$

The study of "explosive behavior," be it finite-time blow-up in evolutionary problems or boundary blow-up in stationary problems, has a long history going back to seminal work by Keller [15] and Osserman [20] in the 1950s; we refer the reader to the papers $[2,4,8,24]$ and the references therein. However, virtually all of the existing literature is concerned with scalar equations. Coupled systems of equations have been attacked only recently; see, for example, $[5,9,10,11,16]$. Due to the lack of variational structure and comparison principles, methods that have proven successful for scalar equations will, in general, fail to be useful for systems, even if the expected results are analogous. For example, our existence and multiplicity result for the problem (1.3) (existence of one large nonnegative solution for any space dimension, existence of a 
second large solution for sufficiently small space dimension) is analogous to a result by McKenna, Reichel, and Walter [17] for a class of scalar equations with variational structure. However, our method of proof is entirely different, and our result appears to be the first of its kind for an elliptic system. We expect that our work, while currently focussed on a very specific problem, will lead to general insights and new methods with potential applications to a much wider class of elliptic and parabolic systems.

The rest of the paper is organized as follows. In section 2 we reduce our problem to the study of a system of first-order ODEs, establish some basic properties of its solutions, and prove the existence and uniqueness of a nonnegative large radial solution for the problem (1.3); Theorem 1.3 is an immediate consequence of Proposition 2.5. Section 3 is devoted to the proof of Theorem 1.4 (existence of a second large radial solution for sufficiently small space dimension), which follows from Proposition 3.1. This section also includes a discussion of numerical experiments, suggesting a sharper version of Theorem 1.4, and observations about a related parameter-dependent fixedpoint equation, leading to a Liouville-type theorem for the Dirichlet problem associated with the elliptic system (1.2). In section 4 we analyze the asymptotic behavior of large radial solutions of (1.3); Theorem 1.5 follows from Proposition 4.1, whose proof relies on dynamical-systems theory applied to an asymptotically autonomous and cooperative ODE system in $\mathbb{R}^{3}$. In an appendix at the end of the paper, we describe a Maple algorithm for the computer-aided construction of a priori bounds needed in the proof of Proposition 3.1.

2. Preliminaries and nonnegative large solutions. Given $N \in \mathbb{N}$ and $R>0$, radially symmetric solutions of the problem (1.3) correspond to solutions of the ODE system

$$
\left\{\begin{array}{l}
v^{\prime \prime}+\frac{N-1}{r} v^{\prime}=\phi, \\
\phi^{\prime \prime}+\frac{N-1}{r} \phi^{\prime}=\left|v^{\prime}\right|^{2}
\end{array} \quad \text { in }(0, R)\right.
$$

with $v^{\prime}(0)=\phi^{\prime}(0)=0$; large solutions are those with $|(v(r), \phi(r))| \rightarrow \infty$ as $r \rightarrow R^{-}$. In view of Remark 1.2, we may impose the initial condition $v(0)=0$. Finding radially symmetric large solutions of the problem (1.3) is therefore equivalent to finding initial conditions $\phi(0)=p$ such that the solution of the Cauchy problem

$$
\begin{cases}v^{\prime \prime}+\frac{N-1}{r} v^{\prime}=\phi, & v(0)=0, v^{\prime}(0)=0, \\ \phi^{\prime \prime}+\frac{N-1}{r} \phi^{\prime}=\left|v^{\prime}\right|^{2}, & \phi(0)=p, \phi^{\prime}(0)=0\end{cases}
$$

exists on the interval $[0, R)$ and "blows up" at $R$.

Despite the singularity at $r=0$ for $N>1$, the Cauchy problem (2.1) is well posed. Indeed, for every $p \in \mathbb{R}$, there exists a unique maximal solution, which depends continuously on $p$ (in the usual sense); see Lemma 2.3 for details.

REMARK 2.1. The scaling property of the elliptic problem (1.3), as described in Remark 1.1, and the well-posedness of (2.1) imply that all solutions of the Cauchy problem with $p>0(p<0)$ are "rescalings" of the solution with $p=1(p=-1)$. Indeed, if $\left(v_{1}, \phi_{1}\right)$ is the maximal solution with initial value $p=1(p=-1)$, then the maximal solution with initial value $p>0(p<0)$ is given by $\left(v_{\lambda}, \phi_{\lambda}\right)$, as defined in Remark 1.1, with $\lambda=|p|^{1 / 4}$. Consequently, if the maximal solution with initial value $p=1(p=-1)$ blows up at $R_{1}$, then the maximal solution with initial value $p>0$ $(p<0)$ blows up at $R_{p}=|p|^{-1 / 4} R_{1}$. 
REMARK 2.2. In light of the preceding remark, it is clear that the elliptic problem (1.3) has large radial solutions, for any given $R>0$, if and only if the solutions of the Cauchy problem (2.1) with $p= \pm 1$ exhibit finite-time blow-up. More precisely, (1.3) has exactly one large radial solution with $v(0)=0$ and $\phi(0)>0$ if and only if the solution of (2.1) with $p=1$ blows up in finite time; (1.3) has exactly one large radial solution with $v(0)=0$ and $\phi(0)<0$ if and only if the solution of (2.1) with $p=-1$ blows up in finite time. In particular, (1.3) cannot have more than two large radial solutions.

The Cauchy problem (2.1) is equivalent to the first-order system

$$
\begin{cases}v^{\prime}=w, & v(0)=0, \\ w^{\prime}+\frac{N-1}{r} w=\phi, & w(0)=0, \\ \phi^{\prime}=\psi, & \phi(0)=p, \\ \psi^{\prime}+\frac{N-1}{r} \psi=w^{2}, & \psi(0)=0 .\end{cases}
$$

Obviously, we can eliminate $v$ and drop the first equation and initial condition; $v$ is recovered from $w$ via antidifferentiation. Furthermore, we may replace the nonnegative integer $N-1$ with a continuous parameter $\mu \in \mathbb{R}_{+}$. Thus, we are led to the Cauchy problem

$$
\begin{cases}w^{\prime}+\frac{\mu}{r} w=\phi, & w(0)=0, \\ \phi^{\prime}=\psi, & \phi(0)=p, \\ \psi^{\prime}+\frac{\mu}{r} \psi=w^{2}, & \psi(0)=0 .\end{cases}
$$

Lemma 2.3. For every $\mu \in \mathbb{R}_{+}$and $p \in \mathbb{R}$, the Cauchy problem (2.2) has a unique maximal, that is, noncontinuable, solution $(w, \phi, \psi) \in C^{1}\left([0, R), \mathbb{R}^{3}\right)$, for some $R \in(0, \infty]$. If $R<\infty$, then $|(w(r), \phi(r), \psi(r))| \rightarrow \infty$ as $r \rightarrow R^{-}$. Moreover, $(w, \phi, \psi)$ depends continuously on $\mu$ and $p$.

Proof. What we claim is that, despite the singularity at $r=0$ in the case $\mu>0$, the Cauchy problem (2.2) has the usual, well-known properties of a regular initialvalue problem in $\mathbb{R}^{3}$. Since we could not find a general result in the literature that would cover our problem, we provide a few remarks on the proof.

Note that the first equation in (2.2) can be written as $\left(r^{\mu} w\right)^{\prime}=r^{\mu} \phi$. Together with the initial condition $w(0)=0$, this is equivalent to the integral equation

$$
w(r)=\int_{0}^{r}\left(\frac{s}{r}\right)^{\mu} \phi(s) d s .
$$

Similarly, the remaining differential equations and initial conditions in (2.2) are equivalent to the integral equations

$$
\phi(r)=p+\int_{0}^{r} \psi(s) d s
$$

and

$$
\psi(r)=\int_{0}^{r}\left(\frac{s}{r}\right)^{\mu} w^{2}(s) d s
$$


Since we have $0<s / r<1$ for $0<s<r$, the "singular term" $(s / r)^{\mu}$ does not cause any difficulties in proving the existence and uniqueness of a solution $(w, \phi, \psi)$ in $C\left([0, \varepsilon], \mathbb{R}^{3}\right)$ of $(2.3)-(2.5)$, for some $\varepsilon>0$, by means of the contraction mapping principle. Clearly, $w, \phi$, and $\psi$ are continuously differentiable on $(0, \varepsilon]$ and satisfy the differential equations and initial conditions in (2.2). In fact, all three components are continuously differentiable on the closed interval $[0, \varepsilon]$. This is obvious for $\phi$, but less so for $w$ and $\psi$. Note, however, that

$$
\begin{aligned}
\lim _{r \rightarrow 0^{+}} w^{\prime}(r) & =\lim _{r \rightarrow 0^{+}}\left(\phi(r)-\frac{\mu}{r} w(r)\right)=p-\mu \lim _{r \rightarrow 0^{+}} \frac{1}{r} \int_{0}^{r}\left(\frac{s}{r}\right)^{\mu} \phi(s) d s \\
& =p-\mu \lim _{r \rightarrow 0^{+}} \frac{1}{r^{\mu+1}} \int_{0}^{r} s^{\mu} \phi(s) d s=p-\mu \lim _{r \rightarrow 0^{+}} \frac{r^{\mu} \phi(r)}{(\mu+1) r^{\mu}} \\
& =p-\mu \lim _{r \rightarrow 0^{+}} \frac{\phi(r)}{\mu+1}=p-\mu \frac{p}{\mu+1}=\frac{p}{\mu+1},
\end{aligned}
$$

where we used l'Hôspital's rule to get the fourth equality. Thus, $w \in C^{1}([0, \varepsilon], \mathbb{R})$ and $w^{\prime}(0)=p /(\mu+1)$. Similarly, one shows that $\psi \in C^{1}([0, \varepsilon], \mathbb{R})$ with $\psi^{\prime}(0)=0$.

Once existence and uniqueness of a local $C^{1}$-solution are established, the remaining claims about maximal continuation and continuous dependence on parameters and initial data can be proved in the same way as for regular initial-value problems.

Lemma 2.4. Let $(w, \phi, \psi) \in C^{1}\left([0, R), \mathbb{R}^{3}\right)$ be the maximal solution of the Cauchy problem (2.2), for some $\mu \in \mathbb{R}_{+}$and $p \in \mathbb{R}$ with $p \neq 0$. Then the function $\phi$ is strictly increasing on $[0, R)$, and $L:=\lim _{r \rightarrow R^{-}} \phi(r)$ is either zero or infinity. In fact,

(a) if $L<\infty$, then $R=\infty$ and $L=0$;

(b) if $L=\infty$, then $R<\infty$ and $w\left(R^{-}\right)=\phi\left(R^{-}\right)=\psi\left(R^{-}\right)=\infty$.

Proof. Taking into account the equations and initial conditions in (2.2), it is easy to see that the function $\Psi(r):=r^{\mu} \psi(r)$ is strictly increasing on $[0, R)$. As a consequence, $\Psi$ (and thus $\psi$ ) is positive on $(0, R)$, and this implies that $\phi$ is strictly increasing on $[0, R)$, with $L:=\lim _{r \rightarrow R^{-}} \phi(r) \in(p, \infty]$.

(a) Assume $L<\infty$, that is, $\phi$ is bounded. By (2.3), w(r) grows at most linearly with $r$, and, by $(2.5), \psi(r)$ grows no faster than $r^{3}$. In particular, $|(w(r), \phi(r), \psi(r))|$ cannot go to infinity in finite time. Thus, $R=\infty$.

Now suppose that $L \neq 0$. If $L>0$, choose a number $r_{0}>0$ such that $\phi(r) \geq L / 2$ for every $r \geq r_{0}$. It follows that

$$
w(r) \geq \int_{0}^{r_{0}}\left(\frac{s}{r}\right)^{\mu} \phi(s) d s+\frac{L}{2} \int_{r_{0}}^{r}\left(\frac{s}{r}\right)^{\mu} d s
$$

for every $r \geq r_{0}$, and we conclude that $\lim _{r \rightarrow \infty} w(r)=\infty$ (note that the last integral is of order $r$ ). If $L<0$, we infer in a similar way that $\lim _{r \rightarrow \infty} w(r)=-\infty$. In any case, we can choose a number $r_{1}>0$ such that $w^{2}(r) \geq 1$ for every $r \geq r_{1}$. As a consequence,

$$
\psi(r) \geq \int_{0}^{r_{1}}\left(\frac{s}{r}\right)^{\mu} w^{2}(s) d s+\int_{r_{1}}^{r}\left(\frac{s}{r}\right)^{\mu} d s
$$

for every $r \geq r_{1}$, and thus $\lim _{r \rightarrow \infty} \psi(r)=\infty$. But this implies

$$
L=\lim _{r \rightarrow \infty} \phi(r)=p+\lim _{r \rightarrow \infty} \int_{0}^{r} \psi(s) d s=\infty,
$$


a contradiction. It follows that $L=0$.

(b) Assume $L=\infty$ and, by way of contradiction, suppose that $R=\infty$. Then there exist $c_{0}, r_{0}>0$ such that $\phi(r) \geq c_{0}$ for every $r \geq r_{0}$, and as in the proof of part (a) it follows that $w(r) \rightarrow \infty$ and $\psi(r) \rightarrow \infty$ as $r \rightarrow \infty$. In particular, we can choose $r_{*}>0$ such that $w(r), \phi(r), \psi(r)>0$ for every $r \geq r_{*}$. Define $\eta:=w \phi \psi$. Then we have

$$
\begin{aligned}
\eta^{\prime} & =\phi^{2} \psi+w \psi^{2}+w^{3} \phi-\frac{2 \mu}{r} w \phi \psi \\
& =Q(w, \phi, \psi) \eta^{13 / 12}-\frac{2 \mu}{r} \eta \quad \text { in }\left[r_{*}, \infty\right)
\end{aligned}
$$

where $Q$ is defined by

$$
Q(x, y, z):=\frac{y^{2} z+x z^{2}+x^{3} y}{(x y z)^{13 / 12}}
$$

for $x, y, z>0$. Note that $Q=Q_{1}+Q_{2}+Q_{3}$, with

$$
Q_{1}:=\frac{y^{2} z}{(x y z)^{13 / 12}}, \quad Q_{2}:=\frac{x z^{2}}{(x y z)^{13 / 12}}, \quad Q_{3}:=\frac{x^{3} y}{(x y z)^{13 / 12}} .
$$

It is easy to see that $Q_{1}^{5} Q_{2}^{4} Q_{3}^{3} \equiv 1$, which implies that $\max \left(Q_{1}, Q_{2}, Q_{3}\right) \geq 1$. Hence, we have $Q(x, y, z) \geq 1$ for all $x, y, z>0$, and (2.6) yields

$$
\eta^{\prime} \geq \eta\left(\eta^{1 / 12}-\frac{2 \mu}{r_{*}}\right) \quad \text { in }\left[r_{*}, \infty\right) .
$$

Recall that $w(r), \phi(r), \psi(r) \rightarrow \infty$ as $r \rightarrow \infty$ and choose $r^{*} \geq r_{*}$ such that $\eta\left(r^{*}\right)>\left(2 \mu / r_{*}\right)^{12}$. Then the maximal solution $\zeta$ of the initial-value problem

$$
\zeta^{\prime}=\zeta\left(\zeta^{1 / 12}-\frac{2 \mu}{r_{*}}\right), \quad \zeta\left(r^{*}\right)=\eta\left(r^{*}\right)
$$

approaches infinity in finite time. But due to (2.7), $\zeta$ is bounded from above by $\eta$ on $\left[r^{*}, \infty\right)$. This is a contradiction, and it follows that $R$ is finite.

In order to prove our last claim, we first note that both $w$ and $\psi$ have (proper or improper) limits as $r \rightarrow R^{-}$. Indeed, since $\phi$ is eventually positive, the function $W(r):=r^{\mu} w(r)$ is eventually increasing, and thus has a limit as $r \rightarrow R^{-}$. As we observed earlier, the same holds for the function $\Psi(r):=r^{\mu} \psi(r)$. Since $R$ is finite, it follows that $w(r)$ and $\psi(r)$, too, have limits as $r \rightarrow R^{-}$. Moreover, since $R$ is finite, all three of the functions $w, \phi, \psi$ would be bounded if one of them were. But $\phi$ is unbounded (by assumption) and thus $w$ and $\psi$ are unbounded as well. Clearly, this implies that $w\left(R^{-}\right)=\phi\left(R^{-}\right)=\psi\left(R^{-}\right)=\infty$.

Proposition 2.5. For every $\mu \in \mathbb{R}_{+}$, the maximal solution of the Cauchy problem (2.2) with $p=1$ blows up in finite time.

Proof. Fix $\mu \in \mathbb{R}_{+}$and let $(w, \phi, \psi) \in C^{1}\left([0, R), \mathbb{R}^{3}\right)$ be the maximal solution of (2.2) with $p=1$. According to Lemma $2.4, \phi$ is increasing and $L:=\lim _{r \rightarrow R^{-}} \phi(r)$ is either zero or infinity. Since $\phi(0)>0$, we have $L=\infty$, and then part (b) of the same lemma implies that $R$ is finite. 
Proof of Theorem 1.3. Thanks to Remark 2.2, the preceding proposition guarantees that the problem (1.3), for arbitrary $N \in \mathbb{N}$ and $R>0$, has exactly one large radial solution $(v, \phi)$ with $v(0)=0$ and $\phi(0)>0$. By Lemma $2.4, \phi$ is a strictly increasing function of the radial variable $r$, and the same then holds for $v$. (Note that, by Lemma 2.4, $\phi(r)$ approaches infinity as $r \rightarrow R^{-}$, and so do $v^{\prime}(r)$ and $\phi^{\prime}(r)$. That the same holds for $v(r)$ is not obvious at this point, but will follow from the blow-up estimates in section 4.)

Figure 1 shows computed profiles of the nonnegative large radial solutions $(v, \phi)$ of the problem (1.3), with $R=1$, for two values of the space dimension $N$ (see Remark 4.4 for comments regarding the numerical method).
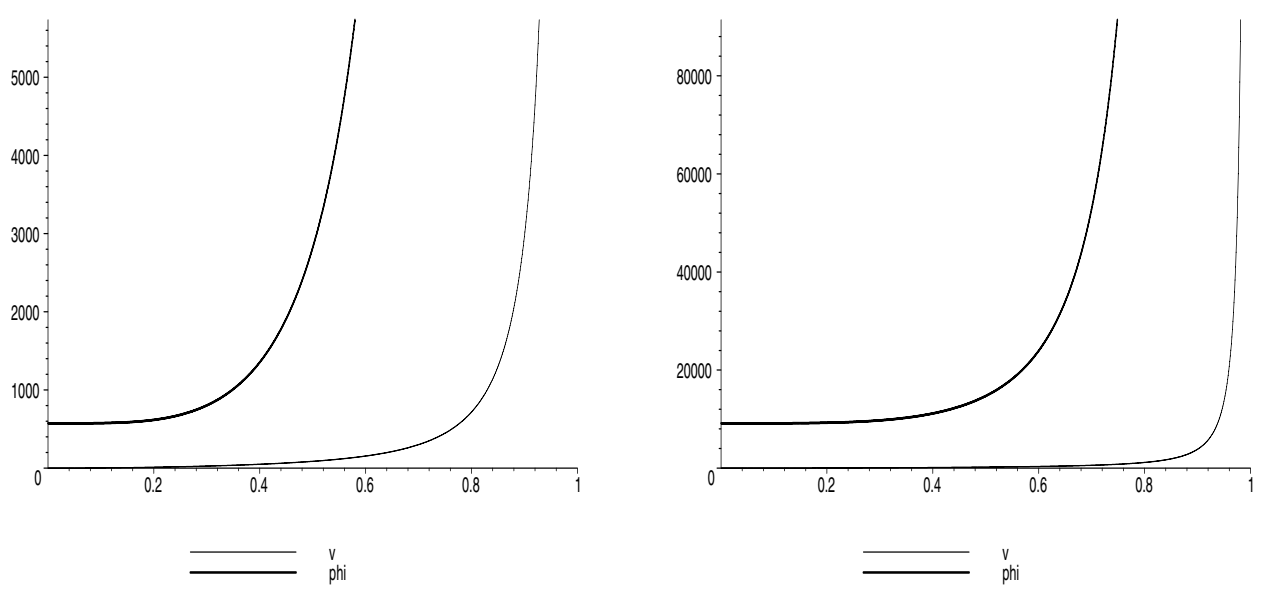

FIG. 1. Large radial solutions $(v, \phi)$ with $v(0)=0$ and $\phi(0)>0$ of problem (1.3) with $R=1$ for $N=1$ (left) and $N=6$ (right).

3. Existence of a second large solution. To prove Theorem 1.4, we need to investigate for which values of $\mu \in \mathbb{R}_{+}$(if any) the maximal solution of the Cauchy problem (2.2) with $p=-1$ blows up in finite time.

It is easy to see that blow-up occurs at least if $\mu \in[0,1]$. Indeed, suppose that $\mu \in \mathbb{R}_{+}$and that the corresponding maximal solution $(w, \phi, \psi)$ of $(2.2)$ with $p=-1$ exists globally. Lemma 2.4 then implies that $\phi(r) \rightarrow 0$ as $r \rightarrow \infty$; thus, $\int_{0}^{\infty} \psi(s) d s=1$, due to (2.4). On the other hand, since $\Psi(r):=r^{\mu} \psi(r)$ is strictly increasing for $r \geq 0$, we have $c:=\Psi(1)>0$ and $\psi(r)=\Psi(r) r^{-\mu} \geq c r^{-\mu}$ for all $r \geq 1$, which implies $\int_{0}^{\infty} \psi(s) d s \geq c \int_{1}^{\infty} s^{-\mu} d s$. Unless $\mu$ is greater than 1 , this shows that $\int_{0}^{\infty} \psi(s) d s=\infty$, and we arrive at a contradiction.

Recalling Remark 2.2, we conclude that the elliptic problem (1.3) has a second large radial solution at least if the space dimension $N$ is 1 or 2 . The following proposition allows us to draw the same conclusion for any space dimension up to and including 10.

Proposition 3.1. For every $\mu \in[0,9]$, the maximal solution of the Cauchy problem (2.2) with $p=-1$ blows up in finite time.

Proof. Fix $\mu \in \mathbb{R}_{+}$and let $(w, \phi, \psi) \in C^{1}\left([0, R), \mathbb{R}^{3}\right)$ be the corresponding maximal solution of (2.2) with $p=-1$. By Lemma 2.4, $R$ is finite if and only if $\phi$ is eventually positive. We will prove the proposition by constructing explicit lower bounds for $\phi$ that are eventually positive if $\mu$ is small enough. 
Clearly, since $\phi$ is an increasing function, we have

$$
\phi(r) \geq \phi(0)=-1=: \underline{\phi}_{0}(r)
$$

for all $r \in[0, R)$. From this, we derive a lower bound for $w$; indeed,

$$
w(r)=\int_{0}^{r}\left(\frac{s}{r}\right)^{\mu} \phi(s) d s \geq \int_{0}^{r}\left(\frac{s}{r}\right)^{\mu} \underline{\phi}_{0}(s) d s=-\frac{r}{\mu+1}=: \underline{w}_{1}(r)
$$

for all $r \in[0, R)$. As long as $w \leq 0$, a lower bound for $w$ yields an upper bound for $\psi$; in particular,

$$
\psi(r)=\int_{0}^{r}\left(\frac{s}{r}\right)^{\mu} w^{2}(s) d s \leq \int_{0}^{r}\left(\frac{s}{r}\right)^{\mu} \underline{w}_{1}^{2}(s) d s=\frac{r^{3}}{(\mu+1)^{2}(\mu+3)}=: \bar{\psi}_{1}(r)
$$

for all $r \in[0, R)$ with $w(r) \leq 0$ (note that $w(r) \leq 0$ implies $\underline{w}_{1} \leq w \leq 0$ on $[0, r]$ ). Next, we find an upper bound for $\phi$, namely,

$$
\phi(r)=-1+\int_{0}^{r} \psi(s) d s \leq-1+\int_{0}^{r} \bar{\psi}_{1}(s) d s=-1+\frac{r^{4}}{4(\mu+1)^{2}(\mu+3)}=: \bar{\phi}_{1}(r),
$$

still valid for all $r \in[0, R)$ with $w(r) \leq 0$. Continuation of this process yields an upper bound for $w$,

$$
w(r) \leq \int_{0}^{r}\left(\frac{s}{r}\right)^{\mu} \bar{\phi}_{1}(s) d s=-\frac{r}{\mu+1}+\frac{r^{5}}{4(\mu+1)^{2}(\mu+3)(\mu+5)}=: \bar{w}_{1}(r),
$$

valid for all $r \in[0, R)$ with $w(r) \leq 0$, and then a lower bound for $\psi$,

$$
\psi(r) \geq \int_{0}^{r}\left(\frac{s}{r}\right)^{\mu} \bar{w}_{1}^{2}(s) d s=: \underline{\psi}_{1}(r),
$$

valid for all $r \in\left[0, r_{0}\right]$, where $r_{0}:=(4(\mu+1)(\mu+3)(\mu+5))^{1 / 4}$ is the unique positive root of $\bar{w}_{1}$ (note that $r_{0}<R$ and $w \leq \bar{w}_{1} \leq 0$ on $\left[0, r_{0}\right]$ ). Finally, we obtain an improved lower bound for $\phi$,

$$
\phi(r) \geq-1+\int_{0}^{r} \underline{\psi}_{1}(s) d s=: \underline{\phi}_{1}(r),
$$

valid for all $r \in\left[0, r_{0}\right]$.

If $\phi_{1}\left(r_{0}\right)$ were nonnegative, the same would hold for $\phi\left(r_{0}\right)$, and this would imply blow-up. Now, $\underline{\phi}_{1}\left(r_{0}\right)$ is easily seen to be a rational function of $\mu$, with a unique positive root $\mu_{1}$, near 3.512 , and positive on the interval $\left[0, \mu_{1}\right)$. Hence, blow-up does occur if $\mu \leq \mu_{1}$.

To improve this result, we iterate the preceding estimates and construct the improved bounds $\underline{w}_{2}, \bar{\psi}_{2}, \bar{\phi}_{2}, \bar{w}_{2}, \underline{\psi}_{2}$, and $\underline{\phi}_{2}$; since $w \leq \bar{w}_{2} \leq \bar{w}_{1} \leq 0$ on $\left[0, r_{0}\right]$, these are still valid on the entire interval $\left[0, r_{0}\right]$. The actual construction is best done with the aid of a computer-algebra system. All the bounds being polynomials, the computations amount to symbolic operations on the coefficients and can be implemented very efficiently. (A more naïve approach, using symbolic antidifferentiation, is likely to fail.) In an appendix at the end of the paper, we describe a Maple implementation of the algorithm. 
Once the improved lower bound $\underline{\phi}_{2}$ for $\phi$ is constructed, we may determine the sign of $\underline{\phi}_{2}\left(r_{0}\right)$; this is once again a rational function of $\mu$, with a unique positive root $\mu_{2}$, near 4.307 , and positive on the interval $\left[0, \mu_{2}\right)$. We conclude that blow-up does occur if $\mu \leq \mu_{2}$.

Any attempt to push this method further by performing another round of estimates turns out to be futile - the computational cost is prohibitive, the gain marginal (the positive root of $\underline{\phi}_{3}\left(r_{0}\right)$ is located near 4.311). Instead, we will extend the lower bound $\underline{\phi}_{2}$ of $\phi$ beyond the interval $\left[0, r_{0}\right]$. To this end, let $r_{1} \in\left(r_{0}, R\right)$ be such that $\phi\left(r_{1}\right) \leq 0$. Then we have $\phi \leq \bar{\phi}_{2}$ on $\left[0, r_{0}\right]$ and $\phi \leq 0$ on $\left[r_{0}, r_{1}\right]$. It follows that for all $r \in\left[r_{0}, r_{1}\right]$,

$$
w(r) \leq \int_{0}^{r_{0}}\left(\frac{s}{r}\right)^{\mu} \bar{\phi}_{2}(s) d s=\left(\frac{r_{0}}{r}\right)^{\mu} \int_{0}^{r_{0}}\left(\frac{s}{r_{0}}\right)^{\mu} \bar{\phi}_{2}(s) d s=\alpha\left(\frac{r_{0}}{r}\right)^{\mu},
$$

where $\alpha:=\bar{w}_{2}\left(r_{0}\right) \leq 0$, and then

$$
\psi(r) \geq \int_{0}^{r_{0}}\left(\frac{s}{r}\right)^{\mu} \bar{w}_{2}^{2}(s) d s+\int_{r_{0}}^{r}\left(\frac{s}{r}\right)^{\mu} \alpha^{2}\left(\frac{r_{0}}{s}\right)^{2 \mu} d s=\beta\left(\frac{r_{0}}{r}\right)^{\mu}-\gamma\left(\frac{r_{0}}{r}\right)^{2 \mu-1},
$$

where $\beta:=\gamma+\underline{\psi}_{2}\left(r_{0}\right), \gamma:=r_{0} \alpha^{2} /(\mu-1)$, and we have implicitly assumed that $\mu \neq 1$ (the case $\mu=1$ will not be needed). Finally, we see that for all $r \in\left[r_{0}, r_{1}\right]$,

$$
\begin{gathered}
\phi(r) \geq-1+\int_{0}^{r_{0}} \underline{\psi}_{2}(s) d s+\int_{r_{0}}^{r}\left(\beta\left(\frac{r_{0}}{s}\right)^{\mu}-\gamma\left(\frac{r_{0}}{s}\right)^{2 \mu-1}\right) d s \\
=\widetilde{\alpha}\left(\frac{r_{0}}{r}\right)^{2(\mu-1)}-\widetilde{\beta}\left(\frac{r_{0}}{r}\right)^{\mu-1}+\widetilde{\gamma},
\end{gathered}
$$

with $\widetilde{\alpha}:=(1 / 2) r_{0} \gamma /(\mu-1), \widetilde{\beta}:=r_{0} \beta /(\mu-1), \widetilde{\gamma}:=(1 / 2) r_{0}(2 \beta-\gamma) /(\mu-1)+\underline{\phi}_{2}\left(r_{0}\right)$, and hence,

$$
0 \geq(\mu-1)^{2} \phi(r) \geq a\left(\frac{r_{0}}{r}\right)^{2(\mu-1)}-b\left(\frac{r_{0}}{r}\right)^{\mu-1}+c,
$$

where

$$
\begin{gathered}
a:=(\mu-1)^{2} \widetilde{\alpha}=\frac{1}{2} r_{0}^{2} \bar{w}_{2}^{2}\left(r_{0}\right), \\
b:=(\mu-1)^{2} \widetilde{\beta}=r_{0}^{2} \bar{w}_{2}^{2}\left(r_{0}\right)+(\mu-1) r_{0} \underline{\psi}_{2}\left(r_{0}\right), \\
c:=(\mu-1)^{2} \widetilde{\gamma}=\frac{1}{2} r_{0}^{2} \bar{w}_{2}^{2}\left(r_{0}\right)+(\mu-1) r_{0} \underline{\psi}_{2}\left(r_{0}\right)+(\mu-1)^{2} \underline{\phi}_{2}\left(r_{0}\right) .
\end{gathered}
$$

The estimate (3.1) holds for every $r \in\left[r_{0}, R\right)$, provided that $\phi(r) \leq 0$, and for any value of $\mu$ (trivially if $\mu=1$ ). The coefficients $a, b$, and $c$ are rational functions of $\mu$ (note that $r_{0}^{4}$ is a polynomial in $\mu$, while $r^{2} \bar{w}_{2}^{2}(r), r \underline{\psi}_{2}(r)$, and $\underline{\phi}_{2}(r)$ are polynomials in $r^{4}$, whose coefficients are rational functions of $\mu$ ); this facilitates their symbolic computation and analysis. In particular, it is easily verified (see the appendix) that $c$ has a unique root, $\bar{\mu} \approx 9.073$, in the interval $(1, \infty)$ and is positive on $(1, \bar{\mu})$. It follows that if $\mu \in(1, \bar{\mu})$, the right-hand side of the inequality (3.1) becomes positive as $r \rightarrow \infty$. But then, the inequality cannot hold for all $r \geq r_{0}$, which implies that $R$ is finite. Since this is already known to be true if $\mu \in[0,1]$, the proposition is proved. 
Remark 3.2. We emphasize that the proof of Proposition 3.1, while relying heavily on the use of a computer-algebra system, does not involve any numerical techniques or floating-point arithmetic. In fact, all the computations amount to symbolic algebra on the coefficients of certain polynomials. The crucial fact that the coefficient $c$ in the estimate (3.1), a rational function of $\mu$, has a unique root, $\bar{\mu} \approx 9.073$, in the interval $(1, \infty)$ can be verified by applying Descartes's rule of signs (and the intermediate-value theorem) to the numerator polynomial (the roots of the denominator polynomial are negative integers). We refer the reader to the appendix for implementation details.

Remark 3.3. The estimates in the proof of Proposition 3.1 involve some deliberate choices, but are in some sense optimal. Of course, it is computationally much less expensive to use the bounds $\bar{w}_{1}, \underline{\psi}_{1}$, and $\underline{\phi}_{1}$ (instead of $\bar{w}_{2}, \underline{\psi}_{2}$, and $\underline{\phi}_{2}$ ) for the "tail estimate" (3.1); also, the estimate itself is simpler in this case, since the coefficient $a$ vanishes. Again, the coefficient $c$ has a unique root in the interval $(1, \infty)$, but it is located near 5.606, leading to a much weaker result. A slight improvement is achieved by using the polynomial bounds $\bar{w}_{1}, \underline{\psi}_{1}$, and $\phi_{1}$ only on the smaller interval $\left[0, \tilde{r}_{0}\right]$ (instead of $\left[0, r_{0}\right]$ ), where $\tilde{r}_{0}:=\left(4(\mu+1)^{2}(\mu+3)\right)^{-1 / 4}$ is the unique positive root of $\bar{\phi}_{1}$. The coefficient $c$ in (3.1) then has a unique positive root near 5.955. Using the improved bounds $\bar{w}_{2}, \underline{\psi}_{2}$, and $\underline{\phi}_{2}$ (as in the proof of Proposition 3.1), but on the smaller interval $\left[0, \tilde{r}_{0}\right]$ (instead of $\left[\overline{0}, r_{0}\right]$ ), yields a coefficient $c$ with a unique positive root near 7.709. It is natural to ask whether it would make sense to construct the bounds $\bar{w}_{3}, \underline{\psi}_{3}$, and $\underline{\phi}_{3}$ before proceeding with the "tail estimate." The answer is negative; in fact, the symbolic computations would require an enormous amount of virtual memory, without leading to a tangibly improved result (the relevant root of the coefficient $c$ in (3.1) is located near 9.170).

REMARK 3.4. The estimates in the proof of Proposition 3.1 yield explicit a priori bounds for the zero $z_{0}$ of $\phi$ (the $\phi$-component of the maximal solution of the Cauchy problem (2.2) with $\mu \in \mathbb{R}_{+}$and $p=-1$ ), assuming that it exists. Clearly, a lower bound is given by $\tilde{r}_{0}:=\left(4(\mu+1)^{2}(\mu+3)\right)^{1 / 4}$, the unique positive root of $\bar{\phi}_{1}$. To establish an upper bound, note that either $z_{0} \leq r_{0}$, where $r_{0}:=(4(\mu+1)(\mu+3)(\mu+5))^{1 / 4}$ is the unique positive root of $\bar{w}_{1}$, or $z_{0}>r_{0}$. In the latter case, which can arise only if $\mu>\mu_{2} \approx 4.307$, (3.1) implies that

$$
a\left(\frac{r_{0}}{z_{0}}\right)^{2(\mu-1)}-b\left(\frac{r_{0}}{z_{0}}\right)^{\mu-1}+c \leq 0
$$

and thus

$$
s_{1} \leq\left(\frac{r_{0}}{z_{0}}\right)^{\mu-1} \leq s_{2}
$$

where $s_{1,2}:=(b \pm \sqrt{d}) /(2 a)$ with $d:=b^{2}-4 a c$ (the discriminant $d$ is positive for $\mu \neq 1$, zero for $\mu=1$ ). As long as $\mu>1$ and $s_{1}$ is positive (which is the case for $1<\mu<\bar{\mu} \approx 9.073)$, it follows that $z_{0} \leq r_{0} s_{1}^{1 /(1-\mu)}$. Hence, an upper bound for $z_{0}$ is given by $r_{0}$ if $0 \leq \mu \leq \mu_{2}$ and by $r_{0} s_{1}^{1 /(1-\mu)}$ if $\mu_{2}<\mu<\bar{\mu}$. A little computation shows that this upper bound may be written, more concisely yet equivalently, as $r_{0} \max \left(1, s_{0}^{1 /(1-\mu)}\right)$, where $s_{0}:=(b-\operatorname{sign}(\mu-1) \sqrt{d}) /(2 a)$. Summarizing, we have

$$
\tilde{r}_{0} \leq z_{0} \leq r_{0} \max \left(1, s_{0}^{1 /(1-\mu)}\right)
$$

for every $\mu \in[0, \bar{\mu})$; the upper bound is a continuous function of $\mu$, positive on $[0, \bar{\mu})$, with a vertical asymptote at $\bar{\mu}$. 
Proof of Theorem 1.4. Due to Remark 2.2, Proposition 3.1 implies that the elliptic problem (1.3), for arbitrary $R>0$, has exactly one large radial solution $(v, \phi)$ with $v(0)=0$ and $\phi(0)<0$ if $\mu=N-1 \leq 9$, that is, if $N \leq 10$. Lemma 2.4 shows that $\phi$ is a strictly increasing function of the radial variable $r$ and crosses zero at a point $z_{0} \in(0, R)$. Hence, the function $W(r):=r^{\mu} w(r)$, with $w=v^{\prime}$, is strictly decreasing for $r<z_{0}$ and strictly increasing for $r>z_{0}$; it crosses zero at a point $z_{1} \in\left(z_{0}, R\right)$. Thus, $v^{\prime}$ is negative on $\left(0, z_{1}\right)$, positive on $\left(z_{1}, R\right)$, and consequently, $v$ is strictly decreasing to a negative minimum at $z_{1}$, strictly increasing thereafter.

Remark 3.5. Numerical evidence suggests that Proposition 3.1 (and with it, Theorem 1.4) may be significantly improved. In fact, there appears to be a number $\tilde{\mu}$, approximately equal to 13.755 , such that the maximal solution of the Cauchy problem (2.2) with $p=-1$ blows up in finite time if and only if $\mu<\tilde{\mu}$. Consequently, we conjecture that the large solution of Theorem 1.4 exists if and only if $N \leq 14$.

Figure 2 depicts computed profiles of the large radial solutions $(v, \phi)$ with $v(0)=0$ and $\phi(0)<0$ of the problem (1.3), with $R=1$, for several values of the space dimension $N$ (see Remark 4.4 for comments on the numerical method). In particular, the solution is shown for $N=10$, the largest space dimension for which we proved its existence, and for $N=14$, the largest space dimension for which we found it numerically. Of course, we can compute the large solution $(v, \phi)$ with $v(0)=0$ and $\phi(0)<0$ of the radial version of (1.3) for every value of $\mu=N-1$, not necessarily integer, up to $\tilde{\mu} \approx 13.755$. As $\mu \rightarrow \tilde{\mu}$, the $\phi$-component of the solution appears to approach $c_{R} \delta_{R}-c_{0} \delta_{0}$, for some positive constants $c_{0}$ and $c_{R}$, where $\delta_{0}$ and $\delta_{R}$ denote the Dirac distributions centered at 0 and $R$, respectively.
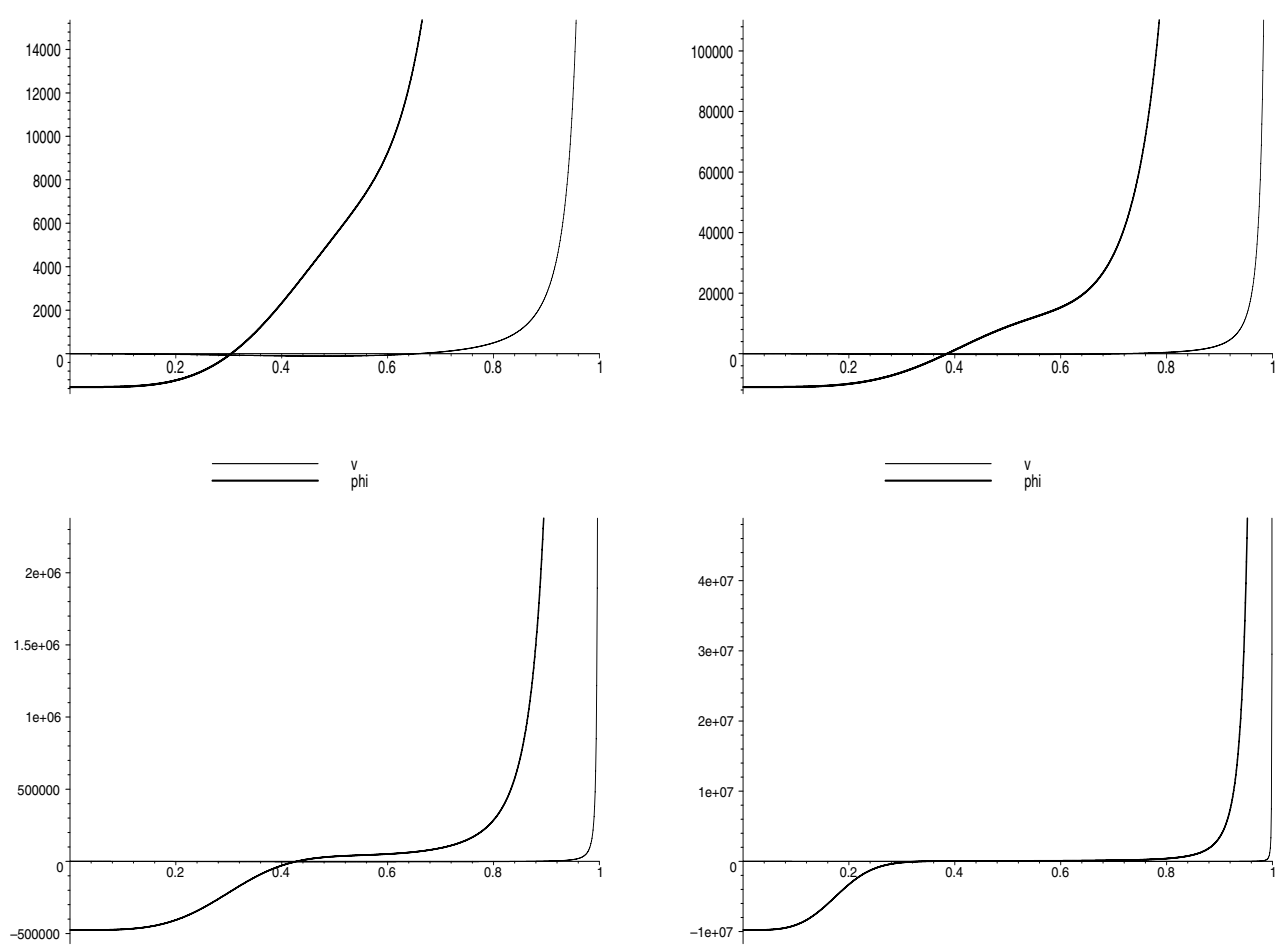

FIG. 2. Large radial solutions $(v, \phi)$ with $v(0)=0$ and $\phi(0)<0$ of problem (1.3) with $R=1$ for $N=1$ (top left), $N=3$ (top right), $N=10$ (bottom left), and $N=14$ (bottom right). 
We will now describe how the question of finite-time blow-up in the Cauchy problem (2.2) can be recast as a question regarding the existence of nontrivial solutions of a related boundary-value problem or, equivalently, a parameter-dependent fixed-point equation. This approach will allow us to exploit standard tools of nonlinear analysis (such as the degree of mapping and bifurcation theory) and to gain some additional information not otherwise available. As a corollary, we will obtain a Liouville-type result (existence of a positive solution) for the Dirichlet problem associated with the elliptic system (1.2), which is of independent interest.

Given $\mu \in \mathbb{R}_{+}$, the maximal solution $(w, \phi, \psi)$ of the Cauchy problem (2.2) with $p=-1$ blows up in finite time if and only if $\phi$ crosses zero at some point $r>0$. Due to the scaling property of the system (see Remark 2.1), this happens if and only if there exists a (necessarily negative and unique) initial value $p$ such that the $\phi$-component of the corresponding maximal solution of (2.2) crosses zero at $r=1$. In other words, the maximal solution of (2.2) with $p=-1$ blows up in finite time if and only if the boundary-value problem

$$
\begin{cases}w^{\prime}+\frac{\mu}{r} w=\phi, & w(0)=0, \\ \phi^{\prime}=\psi, & \phi(1)=0, \\ \psi^{\prime}+\frac{\mu}{r} \psi=w^{2}, & \psi(0)=0\end{cases}
$$

has a (necessarily unique) nontrivial solution.

The problem (3.2) can be written as a parameter-dependent fixed-point equation of the form

$$
u=T(\mu, u)
$$

in $X:=C\left([0,1], \mathbb{R}^{3}\right)$, where $T: \mathbb{R}_{+} \times X \rightarrow X$ is a completely continuous operator, defined by

$$
T(\mu, u)(r):=\left(\int_{0}^{r}\left(\frac{s}{r}\right)^{\mu} \phi(s) d s,-\int_{r}^{1} \psi(s) d s, \int_{0}^{r}\left(\frac{s}{r}\right)^{\mu} w^{2}(s) d s\right),
$$

for $\mu \in \mathbb{R}_{+}, u=(w, \phi, \psi) \in X$, and $r \in[0,1]$. We are interested in the structure of the solution set

$$
\Sigma:=\left\{(\mu, u) \in \mathbb{R}_{+} \times X: u=T(\mu, u)\right\} .
$$

Clearly, $\Sigma$ contains the branch of trivial solutions of (3.3), $\mathbb{R}_{+} \times\{0\}$. Also, as mentioned above, (3.3) cannot have more than one nontrivial solution for any $\mu \in \mathbb{R}_{+}$; hence, the set $\Sigma \backslash\left(\mathbb{R}_{+} \times\{0\}\right)$ is the graph of a function $\mu \mapsto u_{\mu}$. Let $M$ denote the domain of this function, that is,

$$
M:=\left\{\mu \in \mathbb{R}_{+}: u=T(\mu, u) \text { for some } u \in X \backslash\{0\}\right\},
$$

and define

$$
\mu^{*}:=\sup \left(\left\{\mu \in \mathbb{R}_{+}:[0, \mu] \subset M\right\}\right),
$$

with the understanding that $\sup (\emptyset)=0$ and $\sup (A)=\infty$ if $A \subset \mathbb{R}_{+}$is unbounded. 
REMARK 3.6. From the discussion leading to (3.3), it is evident that $M$, as defined in (3.4), coincides with the set of all $\mu \in \mathbb{R}_{+}$for which the maximal solution of the Cauchy problem (2.2) with $p=-1$ blows up in finite time. Thus, $M$ contains the interval $[0,9]$, by Proposition 3.1. The subsequent arguments will prove this once again (we will only need the a priori estimates from Remark 3.4).

REMARK 3.7. We can characterize $M$ as the set of all $\mu \in \mathbb{R}_{+}$for which the $\phi$-component of the maximal solution of $(2.2)$ with $p=-1$ is eventually positive. Since the solution depends continuously on $\mu$, this property is stable under small perturbations of $\mu$. It follows that the set $M$ is open in $\mathbb{R}_{+}$and, hence, $\mu^{*}$ is not an element of $M$. On the other hand, it is clear from the definition of $\mu^{*}$ that $M$ contains the interval $\left[0, \mu^{*}\right)$.

REMARK 3.8. Suppose that $\mu \in M, u_{\mu}=\left(w_{\mu}, \phi_{\mu}, \psi_{\mu}\right)$ is the corresponding nontrivial solution of (3.3), and $u=(w, \phi, \psi)$ is the corresponding maximal solution of the Cauchy problem (2.2) with $p=-1$. Then, due to the scaling property of the problem, $u_{\mu}$ is a "rescaling" of $u$. In fact, denoting the unique zero of $\phi$ by $z_{\mu}$, we have $w_{\mu}(r)=z_{\mu}^{3} w\left(z_{\mu} r\right), \phi_{\mu}(r)=z_{\mu}^{4} \phi\left(z_{\mu} r\right)$, and $\psi_{\mu}(r)=z_{\mu}^{5} \psi\left(z_{\mu} r\right)$, for all $r \in[0,1]$. Since $u=(w, \phi, \psi)$ depends continuously on $\mu$, so does $z_{\mu}$. It follows that the function $\mu \mapsto u_{\mu}$ is continuous as a mapping from $M \subset \mathbb{R}_{+}$into $X$.

Lemma 3.9. Given $\mu \in M$, let $u_{\mu}$ denote the unique nontrivial solution of (3.3). Then $\left\|u_{\mu}\right\|_{\infty} \geq 4(\mu+1)^{2}(\mu+3) \geq 12$. Moreover, there exists a continuous function $\bar{f}:[0, \bar{\mu}) \rightarrow \mathbb{R}_{+}$, with $\bar{\mu} \approx 9.073$, such that $\left\|u_{\mu}\right\|_{\infty} \leq \bar{f}(\mu)$, provided that $\mu \in[0, \bar{\mu})$.

Proof. Fix a number $\mu \in M$, let $u_{\mu}=\left(w_{\mu}, \phi_{\mu}, \psi_{\mu}\right)$ denote the corresponding nontrivial solution of (3.3), and let $u=(w, \phi, \psi)$ denote the corresponding maximal solution of the Cauchy problem (2.2) with $p=-1$. According to Remark 3.8, we have $\phi_{\mu}(r)=z_{\mu}^{4} \phi\left(z_{\mu} r\right)$ for all $r \in[0,1]$, where $z_{\mu}$ is the zero of $\phi$; in particular, $\left|\phi_{\mu}(0)\right|=z_{\mu}^{4}$. Recalling the a priori bounds in Remark 3.4, we obtain the estimate

$$
\left|\phi_{\mu}(0)\right| \geq 4(\mu+1)^{2}(\mu+3) \geq 12
$$

and, furthermore, the existence of a continuous function $f:[0, \bar{\mu}) \rightarrow[1, \infty)$, with $\bar{\mu} \approx 9.073$, such that

$$
\left|\phi_{\mu}(0)\right| \leq 4(\mu+1)(\mu+3)(\mu+5) f(\mu),
$$

provided that $\mu \in[0, \bar{\mu})$. Next, observe that

$$
\left\|\phi_{\mu}\right\|_{\infty}=\left|\phi_{\mu}(0)\right|, \quad\left\|w_{\mu}\right\|_{\infty} \leq \frac{\left|\phi_{\mu}(0)\right|}{\mu+1} \leq\left|\phi_{\mu}(0)\right|,
$$

and

$$
\left\|\psi_{\mu}\right\|_{\infty} \leq \frac{\left|\phi_{\mu}(0)\right|^{2}}{(\mu+1)^{2}(\mu+3)} \leq \frac{1}{3}\left|\phi_{\mu}(0)\right|^{2} ;
$$

since $\left|\phi_{\mu}(0)\right| \geq 12$ by (3.6), this implies that $\left\|\left(w_{\mu}, \phi_{\mu}, \psi_{\mu}\right)\right\|_{\infty} \leq\left|\phi_{\mu}(0)\right|^{2}$. Consequently, we have

$$
\left|\phi_{\mu}(0)\right| \leq\left\|u_{\mu}\right\|_{\infty} \leq\left|\phi_{\mu}(0)\right|^{2},
$$

and now the assertions of the lemma follow from (3.6) and (3.7). 
Proposition 3.10. For every $\mu \in\left[0, \mu^{*}\right)$, with $\mu^{*}$ defined by (3.5), the unique nontrivial solution $u_{\mu}$ of (3.3) has a fixed-point index of -1 . The graph $\mathcal{C}:=\left\{\left(\mu, u_{\mu}\right)\right.$ : $\left.\mu \in\left[0, \mu^{*}\right)\right\}$ is an unbounded, continuous curve in $\mathbb{R}_{+} \times X$, and $\mu^{*}$ is greater than 9.

Proof. We begin by computing the (Leray-Schauder) fixed-point index of the map $T(0, \cdot)$ in $u_{0}$, the nontrivial solution of (3.3) for $\mu=0$. (While the existence of $u_{0}$ was established previously, the following argument will prove it once again.) Inspired by similar reasoning in [1], we define a completely continuous operator $S: \mathbb{R}_{+} \times X \rightarrow X$, with $X:=C\left([0,1], \mathbb{R}^{3}\right)$, by

$$
S(\lambda, u)(r):=\left(\int_{0}^{r} \phi(s) d s,-\int_{r}^{1} \psi(s) d s, \int_{0}^{r}\left(w^{2}(s)+\lambda\right) d s\right)
$$

for $\lambda \in \mathbb{R}_{+}, u=(w, \phi, \psi) \in X$, and $r \in[0,1]$, and consider the parameter-dependent fixed-point problem in $X$,

$$
u=S(\lambda, u)
$$

Note that $S(0, \cdot)=T(0, \cdot)$; that is, if $\lambda=0$, then (3.8) coincides with (3.3) with $\mu=0$.

Now let $\lambda \in \mathbb{R}_{+}$and suppose that $u_{0 \lambda}=\left(w_{0 \lambda}, \phi_{0 \lambda}, \psi_{0 \lambda}\right)$ is a solution of (3.8). Since $\phi_{0 \lambda}^{\prime \prime}=w_{0 \lambda}^{2}+\lambda \geq 0$, the function $\phi_{0 \lambda}$ is convex; thus, $\phi_{0 \lambda}(r) \leq \phi_{0 \lambda}(0)(1-r)$ for all $r \in[0,1]$. Arguing as in the proof of Proposition 3.1, we derive an upper bound for $w_{0 \lambda}$, then a lower bound for $\psi_{0 \lambda}$. Since $\left|\phi_{0 \lambda}(0)\right|=\int_{0}^{1} \psi_{0 \lambda}(s) d s$, the lower bound for $\psi_{0 \lambda}$ yields a quadratic inequality for $\left|\phi_{0 \lambda}(0)\right|$, namely, $\left|\phi_{0 \lambda}(0)\right|^{2}-24\left|\phi_{0 \lambda}(0)\right|+12 \lambda \leq 0$. It follows that $\lambda \leq 12$ and $\left|\phi_{0 \lambda}(0)\right| \leq 24$. This shows that (3.8) does not have any solutions if $\lambda>12$; moreover, the uniform bound on $\left|\phi_{0 \lambda}(0)\right|$ implies a uniform bound on $\left\|u_{0 \lambda}\right\|_{\infty}$.

Choosing a sufficiently large $\rho>0$, we infer that $\operatorname{deg}\left(I d_{X}-S(\lambda, \cdot), B_{\rho}^{X}(0), 0\right)$ is well defined for $\lambda \in \mathbb{R}_{+}$, independent of $\lambda$ (due to homotopy invariance), and in fact equal to zero (since (3.8) has no solutions for $\lambda>12$ ). It follows that

$$
\operatorname{deg}\left(I d_{X}-T(0, \cdot), B_{\rho}^{X}(0), 0\right)=\operatorname{deg}\left(I d_{X}-S(0, \cdot), B_{\rho}^{X}(0), 0\right)=0 .
$$

However, a routine homotopy argument shows that the index of $T(0, \cdot)$ in the trivial fixed point 0 equals 1 . This proves, once again, the existence of the nontrivial fixed point $u_{0}$ (and thereby the fact that $\mu^{*}>0$ ) and shows, more importantly, that the index of $T(0, \cdot)$ in $u_{0}$ is -1 . But then, by homotopy along the continuous curve $\mathcal{C}:=\left\{\left(\mu, u_{\mu}\right): \mu \in\left[0, \mu^{*}\right)\right\}$, the fixed-point index of $T(\mu, \cdot)$ in $u_{\mu}$ is -1 for every $\mu \in\left[0, \mu^{*}\right)$.

We are now in a position to complete the proof of the proposition by applying a Rabinowitz-type argument (see [21]). Suppose that the curve $\mathcal{C}$ is bounded. Then $\mu^{*}<\infty$ and there exists a constant $\rho>0$ such that $\left\|u_{\mu}\right\|_{\infty}<\rho$ for all $\mu \in\left[0, \mu^{*}\right)$. Also, due to Lemma $3.9,\left\|u_{\mu}\right\|_{\infty} \geq 12$ for all $\mu \in\left[0, \mu^{*}\right)$ and, according to Remark 3.7, the equation (3.3) has no nontrivial solution for $\mu=\mu^{*}$. It follows that $\operatorname{deg}\left(I d_{X}-T(\mu, \cdot), B_{\rho}^{X}(0) \backslash \bar{B}_{1}^{X}(0), 0\right)$ is well defined for $\mu \in\left[0, \mu^{*}\right]$, independent of $\mu$, and in fact equal to zero. Clearly, this contradicts the fact that $T(0, \cdot)$ has index -1 in $u_{0}$. It follows that $\mathcal{C}$ is unbounded in $\mathbb{R}_{+} \times X$. In conjunction with Lemma 3.9, according to which $\mathcal{C} \cap([0, \hat{\mu}] \times X)$ is bounded for every $\hat{\mu} \in \mathbb{R}_{+}$with $\hat{\mu}<\bar{\mu} \approx 9.073$, this proves, once again, that $\mu^{*} \geq \bar{\mu}$ and thus $\mu^{*}>9$. 
REMARK 3.11. In light of the numerical evidence described in Remark 3.5, we conjecture that $\mu^{*} \approx 13.755$, that the set $M$ coincides with the interval $\left[0, \mu^{*}\right)$, and that the solution branch $\mathcal{C}$ bifurcates from infinity at $\mu^{*}$.

We conclude this section with a comment on the Dirichlet problem for the elliptic system $(1.2)$ on a ball in $\mathbb{R}^{N}$,

$$
\begin{cases}-\Delta v=\theta & \text { in } B_{R}^{N}(0), \\ -\Delta \theta=|\nabla v|^{2} & \text { in } B_{R}^{N}(0), \\ v=\theta=0 & \text { on } \partial B_{R}^{N}(0) .\end{cases}
$$

Assuming that $R=1$ (due to the scaling property, this entails no loss of generality), there is a one-to-one correspondence between the radial solutions of (3.9) and the solutions of the boundary-value problem (3.2) or the equivalent fixed-point equation (3.3), with $\mu=N-1, \theta=-\phi$, and $v(r)=-\int_{r}^{1} w(s) d s$ for $r \in[0,1]$. Hence, Proposition 3.10 implies that (3.9) has a unique nontrivial radial solution $(v, \theta)$ as long as the space dimension $N$ does not exceed 10; it is easily checked that both components of this solution are positive and strictly decreasing functions of the radial variable $r$.

Corollary 3.12. For every $N \in \mathbb{N}$ with $N \leq 10$ and every $R>0$, the Dirichlet problem (3.9) has a unique nontrivial radially symmetric solution $(v, \theta)$. Both components of this solution are positive and decreasing functions of the radial variable $r$.

Our numerical evidence (see Remark 3.5) suggests that the solution of Corollary 3.12 exists, in fact, if and only if $N \leq 14$. Figure 3 shows computed profiles of this solution for $R=1$ and the two extreme values of the space dimension, $N=1$ and $N=14$. Of course, we can compute the nontrivial solution $(v, \theta)$ of the radial version of (3.9) for any $\mu \in[0, \tilde{\mu})$, with $\tilde{\mu} \approx 13.755$, in place of the integer $N-1$. As $\mu \rightarrow \tilde{\mu}$, the $\theta$-component of the solution appears to approach a multiple of $\delta_{0}$ (the Dirac distribution centered at 0$)$.
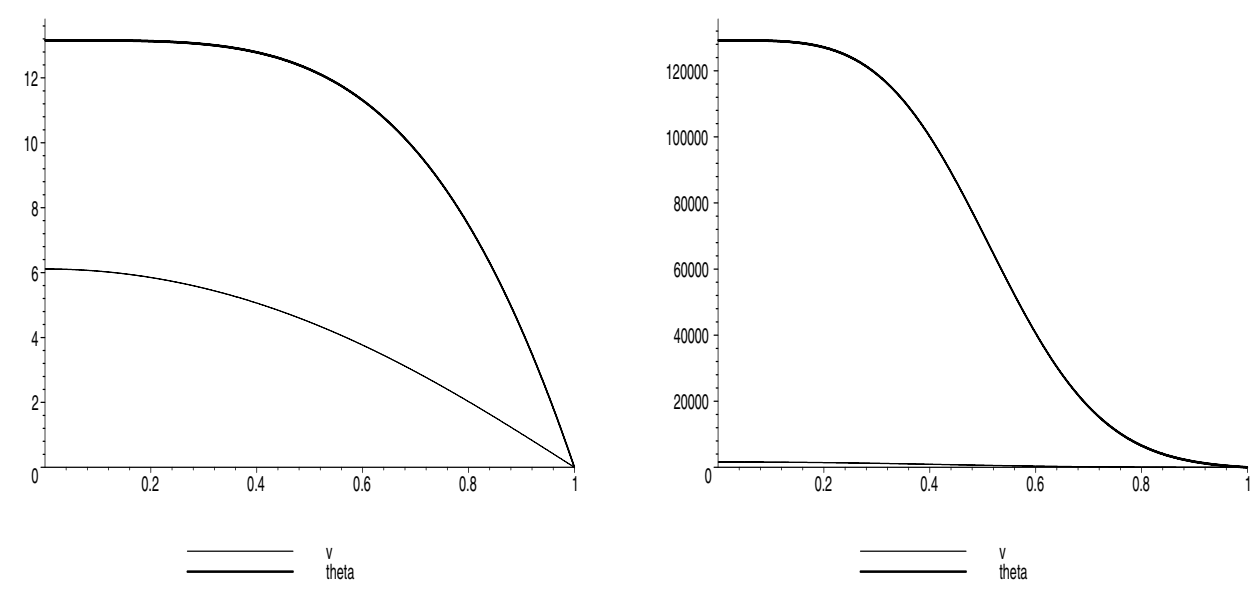

FIG. 3. Positive radial solutions $(v, \theta)$ of the Dirichlet problem (3.9) with $R=1$ for $N=1$ (left) and $N=14$ (right). 
4. Asymptotic behavior. Let $(w, \phi, \psi) \in C^{1}\left([0, R), \mathbb{R}^{3}\right)$ be the maximal solution of the Cauchy problem (2.2) for a given $\mu \in \mathbb{R}_{+}$and $p \in \mathbb{R}$. By Lemma 2.4, we know that $R$ is finite if and only if $\phi(r)$ is eventually positive and that in this case $w(r), \phi(r), \psi(r) \rightarrow \infty$ as $r \rightarrow R^{-}$. In view of the existing literature on boundary blow-up in elliptic equations (see, for example, $[4,24]$ ), it is natural to expect asymptotic behavior of the form $Q /(R-r)^{q}$, with positive constants $Q$ and $q$. In fact, we will prove the following result.

Proposition 4.1. Let $(w, \phi, \psi) \in C^{1}\left([0, R), \mathbb{R}^{3}\right)$ be the maximal solution of the Cauchy problem (2.2), for a given $\mu \in \mathbb{R}_{+}$and $p \in \mathbb{R}$, and suppose that $R$ is finite. Then, as $r \rightarrow R^{-}$,

$$
w(r) \sim \frac{60}{(R-r)^{3}}, \quad \phi(r) \sim \frac{180}{(R-r)^{4}}, \quad \psi(r) \sim \frac{720}{(R-r)^{5}} .
$$

Let us note that if all three of the functions $w, \phi, \psi$ exhibit asymptotic behavior of the form $Q /(R-r)^{q}$, it is easy to see that the constants $Q$ and $q$ are necessarily as above.

We will prove Proposition 4.1 under the assumption that $R=1$; thanks to the scaling property, this entails no loss of generality. The proof will be achieved by analyzing a system of equations derived from (2.2) by a suitable change of variables.

Given any solution $(w, \phi, \psi)$ of $(2.2)$, define functions $\alpha, \beta, \gamma$ by

$$
\alpha(r):=\frac{(1-r)^{3}}{60} w(r), \quad \beta(r):=\frac{(1-r)^{4}}{180} \phi(r), \quad \gamma(r):=\frac{(1-r)^{5}}{720} \psi(r) ;
$$

then $(\alpha, \beta, \gamma)$ is a solution of

$$
\begin{cases}(1-r)\left(\alpha^{\prime}+\frac{\mu}{r} \alpha\right)=3(\beta-\alpha), & \alpha(0)=0, \\ (1-r) \beta^{\prime}=4(\gamma-\beta), & \beta(0)=\frac{p}{180}, \\ (1-r)\left(\gamma^{\prime}+\frac{\mu}{r} \gamma\right)=5\left(\alpha^{2}-\gamma\right), & \gamma(0)=0 .\end{cases}
$$

Just like (2.2), the system (4.1) is singular at $r=0$, but this does not affect the well-posedness of the initial value problem; in addition, (4.1) is singular at $r=1$.

REMARK 4.2. Suppose that $(w, \phi, \psi)$ is the maximal solution of $(2.2)$, for a given $\mu \in \mathbb{R}_{+}$and $p \in \mathbb{R}$, with interval of existence $\left[0, R_{p}\right)$; let $(\alpha, \beta, \gamma)$ be the corresponding solution of (4.1), as defined above. Clearly, if $R_{p}<1$, then $(\alpha, \beta, \gamma)$ ceases to exist before reaching the singularity at $r=1$; in fact, $\alpha(r), \beta(r), \gamma(r) \rightarrow \infty$ as $r \rightarrow R_{p}^{-}$. Also, if $R_{p}>1$, then $(\alpha, \beta, \gamma)$ can be continued beyond the singularity at $r=1$, and $\alpha(r), \beta(r), \gamma(r) \rightarrow 0$ as $r \rightarrow 1^{-}$. Finally, if $R_{p}=1$, then $(\alpha, \beta, \gamma)$ exists up to the singularity at $r=1$, but the behavior near the singularity is not obvious. The assertion of Proposition 4.1 (with $R=1$ ) is that, in this case, $\alpha(r), \beta(r), \gamma(r) \rightarrow 1$ as $r \rightarrow 1^{-}$.

REMARK 4.3. Recall that for every $\mu \in \mathbb{R}_{+}$, there is exactly one initial value $p_{\mu}^{+}>0$ and at most one initial value $p_{\mu}^{-}<0$ such that the maximal solution of (2.2) with $p=p_{\mu}^{ \pm}$blows up at $r=1$. Let $p_{\mu}^{*}$ denote one such value. Due to the scaling property of the problem, all solutions with $\operatorname{sign}(p)=\operatorname{sign}\left(p_{\mu}^{*}\right)$ blow up in finite time. Moreover, there is a one-to-one correspondence between the initial value $p$ and the exit time $R_{p}$ of the solution; in fact, $R_{p}^{4}=p_{\mu}^{*} / p$ (see Remark 2.1). It follows that if $p>p_{\mu}^{*}>0$ or $p<p_{\mu}^{*}<0$, then $R_{p}<1$, and consequently $\alpha(r), \beta(r), \gamma(r) \rightarrow \infty$ as $r \rightarrow R_{p}^{-}$. Also, if $0<p<p_{\mu}^{*}$ or $p_{\mu}^{*}<p<0$, then $R_{p}>1$, and consequently $\alpha(r), \beta(r), \gamma(r) \rightarrow 0$ as $r \rightarrow 1^{-}$. 
REMARK 4.4. The observations in the preceding remark allow us to use a shooting method to numerically approximate $p_{\mu}^{+}$and, if it exists, $p_{\mu}^{-}$, that is, the critical initial values $p$ for which the maximal solution of (2.2), for a given $\mu \in \mathbb{R}_{+}$, blows up at $r=1$. Solving the Cauchy problem (4.1) with $p=p_{\mu}^{ \pm}$, we can then construct the solutions of (2.2) that blow up at $r=1$, and thereby the large radial solutions of the problem (1.3) with $R=1$. All the graphs in the preceding sections were generated in this way (with a suitable rescaling in the case of Figure 3 ).

Our experiments suggest that $p_{\mu}^{-}$exists if and only if $\mu<\tilde{\mu}$, for some number $\tilde{\mu} \approx 13.755$, which, due to the scaling property, must coincide with the number $\mu^{*}$ defined in (3.5). In fact, we find that $p_{\mu}^{-}$is a strictly decreasing function of $\mu$ that approaches $-\infty$ as $\mu \rightarrow \tilde{\mu}$.

To prove Proposition 4.1 for $R=1$, we must show that all three components of the maximal solution $(\alpha, \beta, \gamma)$ of the Cauchy problem (4.1) with $\mu \in \mathbb{R}_{+}$and $p=p_{\mu}^{ \pm}$ converge to 1 as $r \rightarrow 1^{-}$(recall Remarks 4.2 and 4.3). While our numerical experiments leave no doubt about this (see Figure 4 for examples of computed solutions), the proof requires a small detour in dynamical systems; we refer the reader to [22] for terminology and basic properties.
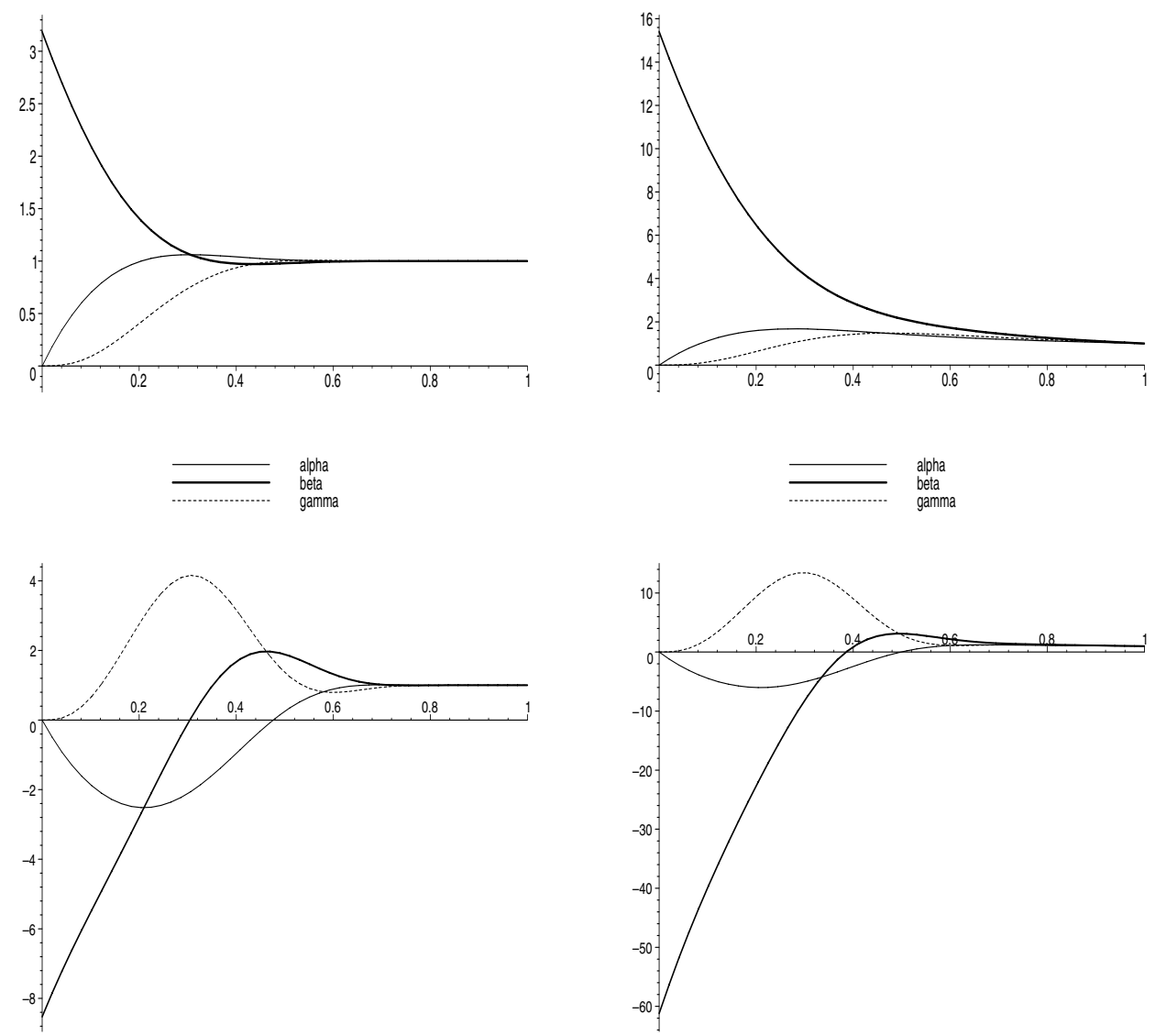

FIG. 4. Critical solutions $(\alpha, \beta, \gamma)$ of problem (4.1) for $\mu=0, p=p_{0}^{+} \approx 180 \times 3.185739467$ (top left), $\mu=2, p=p_{2}^{+} \approx 180 \times 15.39874038$ (top right), $\mu=0, p=p_{0}^{-} \approx-180 \times 8.525812707$ (bottom left), and $\mu=2, p=p_{2}^{-} \approx-180 \times 61.20559852$ (bottom right). 
It is convenient to perform another change of variables in the system (4.1), letting $r=1-e^{-t}$ and $a(t)=\alpha(r), b(t)=\beta(r), c(t)=\gamma(r)$. With this rescaling of the independent variable, (4.1) is equivalent to

$$
\begin{cases}a^{\prime}+\frac{\mu}{e^{t}-1} a=3(b-a), & a(0)=0, \\ b^{\prime}=4(c-b), & b(0)=\frac{p}{180}, \\ c^{\prime}+\frac{\mu}{e^{t}-1} c=5\left(a^{2}-c\right), & c(0)=0 .\end{cases}
$$

Note that the singularity of (4.1) at $r=1$ has been moved to $t=\infty$; moreover, the system (4.2) is autonomous for $\mu=0$ and asymptotically autonomous for $\mu>0$. For notational convenience, we write the system of differential equations in (4.2) as

$$
x^{\prime}+\frac{\mu}{e^{t}-1} E(x)=F(x),
$$

where $x=(a, b, c)$ takes values in $\mathbb{R}^{3}, E: \mathbb{R}^{3} \rightarrow \mathbb{R}^{3}$ is the linear mapping defined by $E(a, b, c):=(a, 0, c)$, and $F: \mathbb{R}^{3} \rightarrow \mathbb{R}^{3}$ is the vector field defined by

$$
F(a, b, c):=\left(3(b-a), 4(c-b), 5\left(a^{2}-c\right)\right) .
$$

REMARK 4.5. For $t>0$ and $a \geq 0$, the system (4.3), with arbitrary $\mu \in \mathbb{R}_{+}$, satisfies the well-known Kamke condition and, thus, a comparison principle (see, for example, [25]). To be precise, let $t_{0}, t_{1} \in[0, \infty]$ with $t_{0}<t_{1}$ and suppose that $x_{1}, x_{2} \in C\left(\left[t_{0}, t_{1}\right), \mathbb{R}^{3}\right) \cap C^{1}\left(\left(t_{0}, t_{1}\right), \mathbb{R}^{3}\right)$. If $x_{1}$ is a subsolution of (4.3) with $a_{1} \geq 0$, if $x_{2}$ is a supersolution of (4.3), and if $x_{1}\left(t_{0}\right) \leq x_{2}\left(t_{0}\right)\left(x_{1}\left(t_{0}\right)<x_{2}\left(t_{0}\right)\right)$, then we have $x_{1}(t) \leq x_{2}(t)\left(x_{1}(t)<x_{2}(t)\right)$ for all $t \in\left[t_{0}, t_{1}\right)$.

By a subsolution (supersolution) of (4.3) we mean a function $x=(a, b, c)$ satisfying the differential inequality obtained from (4.3) by replacing "=" with " $\leq$ " (" $\geq$ "). Also, given vectors $x_{1}, x_{2} \in \mathbb{R}^{3}$, we write $x_{1} \leq x_{2}$ or $x_{2} \geq x_{1}\left(x_{1}<x_{2}\right.$ or $\left.x_{2}>x_{1}\right)$ if the respective inequality holds componentwise, and we call a vector $x \in \mathbb{R}^{3}$ nonnegative (positive) if $x \geq \overline{0}(x>\overline{0})$, where $\overline{0}:=(0,0,0)$.

REMARK 4.6. For every $\lambda \in[0, \infty]$, let $\bar{\lambda}$ denote the vector $(\lambda, \lambda, \lambda)$. For arbitrary $\mu \in \mathbb{R}_{+}, \overline{0}$ is a solution of (4.3), and $\overline{1}$ is a supersolution (a solution if $\mu=0$ ). More generally, for every $\lambda \in[0,1], \bar{\lambda}$ is a supersolution. Furthermore, for every $\lambda \in(1, \infty)$, there exists a number $\tau \in[0, \infty)$, depending only on $\lambda$ and $\mu$, such that $((\lambda+1) / 2, \lambda, \lambda)$ is a subsolution on the interval $(\tau, \infty)$ (where $\tau=0$ if $\mu=0$ ).

Proposition 4.7. For a given $\mu \in \mathbb{R}_{+}$, let $x$ be a nonnegative maximal forward solution of (4.3).

(a) If $x$ is unbounded, then $x$ blows up in finite time and approaches $\bar{\infty}$.

(b) If $x$ is bounded, then $x$ converges to either $\overline{0}$ or $\overline{1}$.

Proof. Fix $\mu \in \mathbb{R}_{+}$and let $x=(a, b, c)$ be a nonnegative maximal forward solution of the system (4.3).

(a) Suppose that $x=(a, b, c)$ is unbounded. First we will show that $b$ is unbounded. By way of contradiction, suppose that $b \leq b_{0}$ for some positive constant $b_{0}$. Then we have

$$
a^{\prime} \leq a^{\prime}+\frac{\mu}{e^{t}-1} a=3(b-a) \leq 3\left(b_{0}-a\right),
$$

which implies that $a$ is bounded. A similar argument then shows that $c$ is bounded as well, and this contradicts the unboundedness of $x=(a, b, c)$. Thus, $b$ is unbounded. 
Now fix a number $p_{0}>0$ such that the solution $\left(w_{0}, \phi_{0}, \psi_{0}\right)$ of the initial-value problem (2.2) with $p=p_{0}$ blows up at a point $R_{0}<1$. The corresponding solution $x_{0}=\left(a_{0}, b_{0}, c_{0}\right)$ of the initial-value problem $(4.2)$ then blows up at $-\log \left(1-R_{0}\right)$. Recall that the $b$-component of the trajectory $x=(a, b, c)$ is unbounded and choose a point $\tau$ in the interval of existence of $x$ such that $b(\tau) \geq p_{0} / 180$; define $\tilde{x}:=x(\tau+\cdot)$. Then we have

$$
\tilde{x}^{\prime}+\frac{\mu}{e^{t}-1} E(\tilde{x}) \geq \tilde{x}^{\prime}+\frac{\mu}{e^{\tau+t}-1} E(\tilde{x})=F(\tilde{x})
$$

and

$$
\tilde{x}(0)=x(\tau)=(a(\tau), b(\tau), c(\tau)) \geq\left(0, \frac{p_{0}}{180}, 0\right)=x_{0}(0) .
$$

Thanks to the comparison principle in Remark 4.5 , it follows that $\tilde{x} \geq x_{0}$. In particular, $\tilde{x}$ blows up in finite time, and then so does $x$. By reasoning as in the proof of Lemma 2.4(b), it is now easy to verify that all three components of $x$ approach infinity.

(b) Suppose that $x$ is bounded. First, consider the autonomous case, $\mu=0$. The vector field $F$ is cooperative in the half-space $a \geq 0$ and, in particular, in the nonnegative cone $\mathbb{R}_{+}^{3}$; moreover, $\operatorname{div}(F) \equiv-12$ and $F$ has exactly two zeros, at $\overline{0}$ and $\overline{1}$. Thus, $F$ generates a monotone, volume-contracting semiflow $\Phi$ in $\mathbb{R}_{+}^{3}$, with exactly two equilibria, at $\overline{0}$ and $\overline{1}$. The equilibrium at $\overline{0}$ is a stable node; the equilibrium at $\overline{1}$ is a saddle point with a two-dimensional stable manifold (the eigenvalues are 1 and $-13 / 2 \pm i \sqrt{71} / 2)$.

Moreover, the system can be embedded into a cooperative system in all of $\mathbb{R}^{3}$ by replacing the nonlinear term $a^{2}$ in the third component of the vector field $F$ with $a|a|$; the extended system still has negative divergence, and it has equilibria at $\overline{0}$ and $\pm \overline{1}$. Morris Hirsch proved (see [13, Theorem 1]) that every compact limit set of a cooperative or competitive system in $\mathbb{R}^{3}$ is either a cycle or contains an equilibrium. Another result of Hirsch's (see [12, Theorem 7]) guarantees that a cooperative system in $\mathbb{R}^{3}$ with negative divergence cannot have any cycles. Moreover, for our particular system, it is easy to see that any compact limit set containing one of the equilibria is in fact a singleton. Combining these results we infer that every bounded (forward or backward) trajectory of the system converges. In particular, the trajectory $x$ converges to either $\overline{0}$ or $\overline{1}$.

Now consider the nonautonomous case, $\mu>0$. As we observed before, the system (4.3) is asymptotically autonomous. An old result of Markus [18] implies that the $\omega$-limit set $K$ of the trajectory $x$ is a nonempty compact and connected subset of $\mathbb{R}_{+}^{3}$; moreover, $\operatorname{dist}(x(t), K) \rightarrow 0$ as $t \rightarrow \infty$, and $K$ is invariant under the semiflow $\Phi$ of the autonomous limit system, that is, (4.3) with $\mu=0$. A more recent result by Mischaikow, Smith, and Thieme (see [19, Theorem 1.8]) implies that $K$ is also chain-recurrent under $\Phi$.

We claim that $K \subset\{\overline{0}, \overline{1}\}$. By way of contradiction, suppose there is a point $z \in K \backslash\{\overline{0}, \overline{1}\}$. In light of what we proved for the autonomous case, since $K$ is compact and $\Phi$-invariant, the $\Phi$-trajectory through $z$ must converge, both forward and backward in time. Backward in time, it can only converge to $\overline{1}$ (since $\overline{0}$ is stable). Thus, $z$ belongs to the unstable manifold of $\overline{1}$, and it follows that, forward in time, the $\Phi$-trajectory through $z$ can only converge to $\overline{0}$. Hence, $K$ consists of the two equilibria, $\overline{0}$ and $\overline{1}$, and a heteroclinic orbit connecting the two; such a set is obviously not chain-recurrent. The contradiction proves that $K \subset\{\overline{0}, \overline{1}\}$. In fact, since $K$ is nonempty and connected, we have either $K=\{\overline{0}\}$ or $K=\{\overline{1}\}$; that is, $x$ converges to either $\overline{0}$ or $\overline{1}$. 
Corollary 4.8. Let $\mu \in \mathbb{R}_{+}$and $p \in \mathbb{R}$ be such that the maximal solution $(w, \phi, \psi)$ of the Cauchy problem (2.2) blows up at $r=1$. Then the maximal solution $(a, b, c)$ of $(4.2)$ converges to $\overline{1}$.

Proof. Under the assumptions of the corollary, $(w, \phi, \psi)$ approaches $\bar{\infty}$ at $r=1$; thus, the corresponding solution $(\alpha, \beta, \gamma)$ of (4.1) is eventually positive and exists on $[0,1)$ (see Remark 4.2). This means that $x=(a, b, c)$ is eventually positive and exists on $[0, \infty)$. By part (a) of Proposition 4.7, it follows that $x$ is bounded, and then part (b) of the same proposition implies that $x$ converges to either $\overline{0}$ or $\overline{1}$. Now suppose that $x(t) \rightarrow \overline{0}$ as $t \rightarrow \infty$ and choose $t_{0} \in(0, \infty)$ such that $\overline{0}<x\left(t_{0}\right)<\overline{1}$. Since the solution of (4.2) depends continuously on $p$, we can find a value $\tilde{p}$ close to $p$, with $|\tilde{p}|>|p|$ such that the corresponding maximal solution $\tilde{x}$ of (4.2) exists at $t=t_{0}$ and satisfies $\overline{0}<\tilde{x}\left(t_{0}\right)<\overline{1}$. Since $\overline{0}$ is a solution and $\overline{1}$ is a supersolution of (4.3), the comparison principle in Remark 4.5 implies that $\overline{0}<\tilde{x}(t)<\overline{1}$ for all $t \geq t_{0}$, as long as $\tilde{x}$ exists. From Remark 4.3 , however, we know that $\tilde{x}$ goes to $\bar{\infty}$ (in finite time). This contradiction proves that $x$ does not converge to $\overline{0}$, and therefore it must converge to $\overline{1}$.

Proof of Proposition 4.1. Due to the scaling property of the system (2.2) (see Remark 2.1), it suffices to prove the assertion of the proposition for $R=1$; in this case, it is an immediate consequence of Corollary 4.8 (see Remark 4.2).

Proof of Theorem 1.5. Since every large radial solution $(v, \phi)$ of the problem (1.3), for a given $R>0$, corresponds to a solution $(w, \phi, \psi)$ of $(2.2)$ that blows up at $R$, the asymptotic behavior of $\phi$ is clear from Proposition 4.1. Moreover, the asymptotic behavior of $v$, given by $v(r)=\int_{0}^{r} w(s) d s$ for $0 \leq r<R$, follows readily from that of $w$.

In closing, we note that Hirsch's results on cooperative systems in $\mathbb{R}^{3}$ (see [12, 13]) allow us to completely describe the dynamics of the monotone, volume-contracting semiflow $\Phi$ in $\mathbb{R}_{+}^{3}$, induced by the vector field $F$. First, it is easily verified that $\Phi$ is, in fact, strongly monotone (even though $F$ is irreducible only for $a>0$ ). As shown in the first part of the proof of Proposition 4.7(b), Hirsch's results imply that every forward trajectory of $\Phi$ either converges to $\overline{0}$ (a stable node) or to $\overline{1}$ (a saddle point), or it approaches $\bar{\infty}$, necessarily in finite time. Clearly, both $\overline{0}$ and $\bar{\infty}$ are stable attractors. In fact, using the sub- and super-solutions constructed in Remark 4.6, we see that the open order intervals $(\overline{0}, \overline{1})$ and $(\overline{1}, \bar{\infty})$ are positively invariant and contained in the basins of attraction of $\overline{0}$ and $\bar{\infty}$, respectively. The two basins of attraction are separated by the (two-dimensional) stable manifold $W_{s}(\overline{1})$ of the saddle point. The (one-dimensional) unstable manifold $W_{u}(\overline{1})$ has a positive tangent vector at $\overline{1}$, which implies that $W_{u}(\overline{1}) \backslash\{\overline{1}\}$ is contained in the union of the order intervals $(\overline{0}, \overline{1})$ and $(\overline{1}, \bar{\infty})$. Thus, every forward trajectory on $W_{u}(\overline{1}) \backslash\{\overline{1}\}$ either converges to $\overline{0}$ or approaches $\bar{\infty}$. It follows that $W_{u}(\overline{1}) \backslash\{\overline{1}\}$ consists of two heteroclinic orbits connecting $\overline{1}$ to $\overline{0}$ and $\bar{\infty}$, respectively.

Appendix. The following algorithm allows the construction of increasing sequences of polynomial lower bounds and decreasing sequences of polynomial upper bounds for the maximal solution $(w, \phi, \psi)$ of the Cauchy problem (2.2) with $p=-1$ and arbitrary $\mu \in \mathbb{R}_{+}$. The bounds being polynomials, the computations amount to symbolic algebra on the coefficients. We used Maple (Version 9.5) to perform these computations; the relevant commands are provided below.

Given a polynomial $P$ in $r$ (whose coefficients are rational functions of another variable), the command

$$
L:=\text { normal (CoefficientList }(P, r)) \text { : }
$$


generates the list of coefficients of $P$ (in increasing order, starting with the zeroorder term) and writes each coefficient in "normal form" (that is, as a quotient of polynomials). The command

$$
\mathrm{n}:=\operatorname{nops}(\mathrm{L}):
$$

gives the number of entries in the list $L$; that is, $n-1$ is the degree of $P$.

Algorithm: Construction of Polynomial Bounds.

0 . Specify a lower (or an upper) bound $\phi_{0}$ for $\phi\left(\phi_{0}\right.$ a suitable polynomial in $r$ ).

$$
\begin{aligned}
& \text { with (PolynomialTools) : } \\
& \text { phi0:=-1; \# Other choices are possible. }
\end{aligned}
$$

1. Compute a lower (upper) bound $w_{0}$ for $w: w_{0}:=\int_{0}^{r}(s / r)^{\mu} \phi_{0}(s) d s$.

$$
\begin{gathered}
\mathrm{L}:=\operatorname{normal}(\text { CoefficientList }(\operatorname{phi}, \mathrm{r})): \mathrm{n}:=\operatorname{nops}(\mathrm{L}): \\
\text { w0 }:=\operatorname{sum}\left(\mathrm{L}[\mathrm{i}] * \mathrm{r}^{\wedge} \mathrm{i} /(\mathrm{mu}+\mathrm{i}), \mathrm{i}=1 \ldots \mathrm{n}\right) ;
\end{gathered}
$$

2. Compute an upper (lower) bound $\psi_{0}$ for $\psi$, valid as long as $\max \left(w, w_{0}\right) \leq 0$ : $\psi_{0}:=\int_{0}^{r}(s / r)^{\mu} w_{0}^{2}(s) d s$.

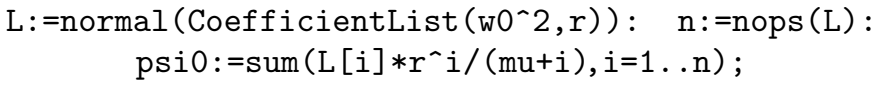

3. Compute an upper (lower) bound $\phi_{0}$ for $\phi: \phi_{0}:=-1+\int_{0}^{r} \psi_{0}(s) d s$.

$$
\begin{gathered}
\mathrm{L}:=\operatorname{normal}(\text { CoefficientList }(\operatorname{psi} 0, \mathrm{r})): \mathrm{n}:=\operatorname{nops}(\mathrm{L}): \\
\operatorname{phi0}:=-1+\operatorname{sum}\left(\mathrm{L}[\mathrm{i}] * \mathrm{r}^{\wedge} \mathrm{i} / \mathrm{i}, \mathrm{i}=1 \ldots \mathrm{n}\right) ;
\end{gathered}
$$

4. Return to Step 1 (or proceed to "Computation of coefficient $c$ " below).

Starting with the trivial lower bound $\underline{\phi}_{0}:=-1$ for $\phi$, the algorithm produces the bounds $\underline{w}_{1}, \bar{\psi}_{1}, \bar{\phi}_{1}, \bar{w}_{1}, \underline{\psi}_{1}, \underline{\phi}_{1}, \ldots$, referred to in the proof of Proposition 3.1 ; these bounds are valid on the interval $\left[0, r_{0}\right]$, where $r_{0}:=(4(\mu+1)(\mu+3)(\mu+5))^{1 / 4}$ is the unique positive root of $\bar{w}_{1}$ (note that for all $k \in \mathbb{N}, \underline{w}_{1} \leq \underline{w}_{k} \leq w \leq \bar{w}_{k} \leq \bar{w}_{1} \leq 0$ on $\left.\left[0, r_{0}\right]\right)$.

Now suppose that $w_{0}, \psi_{0}$, and $\phi_{0}$ have been constructed according to Steps 1-3 of the algorithm, starting with an initially negative upper bound for $\phi$. Then we have $w \leq w_{0} \leq 0, \psi \geq \psi_{0} \geq 0$, and $\phi \geq \phi_{0}$ on some interval $\left[0, r_{0}\right]$ with $r_{0}>0$. The arguments in the proof of Proposition 3.1 show that the following "tail estimate" holds for every $r>r_{0}$, provided that $\phi(r) \leq 0$ :

$$
0 \geq(\mu-1)^{2} \phi(r) \geq a\left(\frac{r_{0}}{r}\right)^{2(\mu-1)}-b\left(\frac{r_{0}}{r}\right)^{\mu-1}+c
$$

where

$$
\begin{aligned}
a & :=\frac{1}{2} r_{0}^{2} w_{0}^{2}\left(r_{0}\right), \quad b:=r_{0}^{2} w_{0}^{2}\left(r_{0}\right)+(\mu-1) r_{0} \psi_{0}\left(r_{0}\right), \\
c & :=\frac{1}{2} r_{0}^{2} w_{0}^{2}\left(r_{0}\right)+(\mu-1) r_{0} \psi_{0}\left(r_{0}\right)+(\mu-1)^{2} \phi_{0}\left(r_{0}\right) .
\end{aligned}
$$

If we use the bounds $w_{0}=\bar{w}_{k}, \psi_{0}=\underline{\psi}_{k}$, and $\phi_{0}=\underline{\phi}_{k}$, for some $k \in \mathbb{N}$, and choose $r_{0}$ to be the positive root of $\bar{w}_{1}$ (or the positive root of $\bar{\phi}_{1}$ ), the coefficients $a, b, c$, and various other relevant quantities, such as $\phi_{0}\left(r_{0}\right)$, are rational functions of $\mu$; in fact, $r_{0}^{4}$ is a polynomial in $\mu$, while $r^{2} w_{0}^{2}(r), r \psi_{0}(r)$, and $\phi_{0}(r)$ are polynomials in $r^{4}$, 
whose coefficients are rational functions of $\mu$. This facilitates the symbolic computation of these quantities and allows us to locate their positive roots by inspecting the coefficients of the respective numerator polynomials (the roots of the denominator polynomials are negative integers). Recall that finding the positive roots of $c$, in particular, is a crucial step in the proof of Proposition 3.1.

Algorithm: Computation of Coefficient c. 0 . Specify $\rho:=r_{0}^{4}(\rho$ a suitable polynomial in $\mu$ ).

$$
\begin{gathered}
\text { rho: }:=4 *(\mathrm{mu}+1) *(\mathrm{mu}+3) *(\mathrm{mu}+5): \\
\text { \# Alternatively: rho: }=4 *(\mathrm{mu}+1)^{\wedge} 2 *(\mathrm{mu}+3):
\end{gathered}
$$

1. Compute coefficients of $(1 / 2) r^{2} w_{0}^{2}(r)+(\mu-1) r \psi_{0}(r)+(\mu-1)^{2} \phi_{0}(r)$.

$$
\begin{gathered}
\mathrm{P}:=\mathrm{r}^{\wedge} 2 * \mathrm{w}^{\wedge} 2 / 2+(\mathrm{mu}-1) * r * \mathrm{psi0}+(\mathrm{mu}-1) \wedge 2 * \mathrm{phi0}: \\
\mathrm{L}:=\operatorname{normal}(\text { CoefficientList }(\mathrm{P}, \mathrm{r})): \mathrm{n}:=(\operatorname{nops}(\mathrm{L})-1) / 4+1:
\end{gathered}
$$

2. Compute coefficient $c=c_{1} / c_{2}$, with numerator polynomial $c_{1}$ and denominator polynomial $c_{2}$.

$$
\begin{gathered}
c:=\operatorname{normal}\left(\operatorname{sum}\left(L[4 * i-3] * r h 0^{\wedge}(i-1), i=1 \ldots \mathrm{n}\right)\right): \\
\quad c 1:=\operatorname{sort}(\operatorname{numer}(c)) ; \quad c 2:=\operatorname{denom}(c) ;
\end{gathered}
$$

If we choose $w_{0}=\bar{w}_{2}, \psi_{0}=\underline{\psi}_{2}, \phi_{0}=\underline{\phi}_{2}$, and $\rho=4(\mu+1)(\mu+3)(\mu+5)$, as in the proof of Proposition 3.1, then the numerator polynomial $c_{1}$ of $c$ has exactly two positive roots (by Descartes's rule of signs and the intermediate-value theorem), one near 0.747 and the other near 9.073 .

Acknowledgments. This project was started while M. Lazzo and P. G. Schmidt were visiting the Universidad Politécnica de Madrid and the Universidad Complutense de Madrid; both wish to express their gratitude to J. F. Padial and J. I. Díaz for their generous support. Most of the work was done while M. Lazzo was visiting Auburn University; she would like to thank the faculty and staff of the Department of Mathematics and Statistics for their kind hospitality. Both M. Lazzo and P. G. Schmidt are grateful for stimulating discussions with W. Shen and G. Hetzer at Auburn. Finally, the authors wish to thank the anonymous referees for their constructive criticism and valuable suggestions.

\section{REFERENCES}

[1] C. Azizien, P. Clément, And E. Mitidieri, Existence and a priori estimates for positive solutions of p-Laplace equations, J. Differential Equations, 184 (2002), pp. 422-442.

[2] C. Bandle and H. Brunner, Blowup in diffusion equations: A survey, J. Comput. Appl. Math., 97 (1998), pp. 3-22.

[3] C. Bandle, G. Díaz, And J. I. Díaz, Solutions d'équations de réaction-diffusion non linéaires explosant au bord parabolique, C. R. Acad. Sci. Paris Sér. I Math., 318 (1994), pp. 455-460.

[4] C. Bandle And M. Marcus, "Large" solutions of semilinear elliptic equations: Existence, uniqueness and asymptotic behaviour, J. Anal. Math., 58 (1992), pp. 9-24.

[5] F.-C. Şt. Cîrstea And V. D. RĂdulescu, Entire solutions blowing up at infinity for semilinear elliptic systems, J. Math. Pures Appl., 81 (2002), pp. 827-846.

[6] M. G. Crandall, P. L. Lions, And P. E. Souganidis, Maximal solutions and universal bounds for some partial differential equations of evolution, Arch. Rational Mech. Anal., 105 (1989), pp. 163-190.

[7] J. I. DíAz, Obstruction and some approximate controllability results for the Burgers equation and related problems, in Control of Partial Differential Equations and Applications (Proc. Int. Conf., Laredo, 1994), Lecture Notes in Pure and Appl. Math. 174, E. Casas, ed., Marcel Dekker, New York, 1996, pp. 63-76. 
[8] G. DíAz AND R. Letelier, Explosive solutions of quasilinear elliptic equations: Existence and uniqueness, Nonlinear Anal., 20 (1993), pp. 97-125.

[9] J. García-Melián and J. D. Rossi, Boundary blow-up solutions to elliptic systems of competitive type, J. Differential Equations, 206 (2004), pp. 156-181.

[10] J. García-Melián and A. SuÁrez, Existence and uniqueness of positive large solutions to some cooperative elliptic systems, Adv. Nonlinear Stud., 3 (2003), pp. 193-206.

[11] M. Ghergu And V. D. RĂDulescu, Explosive solutions of semilinear elliptic systems with gradient term, RACSAM Rev. R. Acad. Cien. Exactas Fis. Nat. Ser. A. Mat., 97 (2003), pp. $467-475$.

[12] M. W. HIRSCH, Systems of differential equation that are competitive or cooperative. V. Convergence in 3-dimensional systems, J. Differential Equations, 80 (1989), pp. 94-106.

[13] M. W. HiRsch, Systems of differential equations that are competitive or cooperative. IV: Structural stability in three-dimensional systems, SIAM J. Math. Anal., 21 (1990), pp. 12251234 .

[14] S. KAmin AND L. VÉRON, Existence and uniqueness of the very singular solution of the porous media equation with absorption, J. Anal. Math., 51 (1988), pp. 245-258.

[15] J. B. Keller, On solutions of $\Delta u=f(u)$, Comm. Pure Appl. Math., 10 (1957), pp. 503-510.

[16] A. V. LAIR AND A. W. WoOd, Existence of entire large positive solutions of semilinear elliptic systems, J. Differential Equations, 164 (2000), pp. 380-394.

[17] P. J. McKenna, W. Reichel, And W. Walter, Symmetry and multiplicity for nonlinear elliptic differential equations with boundary blow-up, Nonlinear Anal., 28 (1997), pp. 12131225 .

[18] L. Markus, Asymptotically autonomous differential systems, in Contributions to the Theory of Nonlinear Oscillations 3, Ann. of Math. Stud. 36, S. Lefschetz, ed., Princeton University Press, Princeton, NJ, 1956, pp. 17-29.

[19] K. Mischaikow, H. Smith, and H. R. Thieme, Asymptotically autonomous semiflows: Chain recurrence and Lyapunov functions, Trans. Amer. Math. Soc., 347 (1995), pp. 1669-1685.

[20] R. Osserman, On the inequality $\Delta u \geq f(u)$, Pacific J. Math., 7 (1957), pp. 1641-1647.

[21] P. H. Rabinowitz, Some global results for nonlinear eigenvalue problems, J. Funct. Anal., 7 (1971), pp. 487-513.

[22] H. L. Smith, Monotone Dynamical Systems: An Introduction to the Theory of Competitive and Cooperative Systems, Math. Surveys Monogr. 41, AMS, Providence, RI, 1995.

[23] J. L. VÁzQUEZ AND M. WALÍAs, Existence and uniqueness of solutions of diffusion-absorption equations with general data, Differential Integral Equations, 7 (1994), pp. 15-36.

[24] L. VÉron, Semilinear elliptic equations with uniform blow-up on the boundary, J. Anal. Math., 59 (1992), pp. 231-250.

[25] W. WALter, Differential and Integral Inequalities, Springer-Verlag, Berlin, 1970. 